TRANSACTIONS OF THE

AMERICAN MATHEMATICAL SOCIETY

Volume 357, Number 8, Pages 3051-3082

S 0002-9947(04)03494-4

Article electronically published on July 16, 2004

\title{
DESCENT REPRESENTATIONS AND MULTIVARIATE STATISTICS
}

\author{
RON M. ADIN, FRANCESCO BRENTI, AND YUVAL ROICHMAN
}

\begin{abstract}
Combinatorial identities on Weyl groups of types $A$ and $B$ are derived from special bases of the corresponding coinvariant algebras. Using the Garsia-Stanton descent basis of the coinvariant algebra of type $A$ we give a new construction of the Solomon descent representations. An extension of the descent basis to type $B$, using new multivariate statistics on the group, yields a refinement of the descent representations. These constructions are then applied to refine well-known decomposition rules of the coinvariant algebra and to generalize various identities.
\end{abstract}

\section{INTRODUCTION}

1.1. Outline. This paper studies the interplay between representations of classical Weyl groups of types $A$ and $B$ and combinatorial identities on these groups. New combinatorial statistics on these groups are introduced, which lead to a new construction of representations. The Hilbert series which emerge give rise to multivariate identities generalizing known ones.

The set of elements in a Coxeter group having a fixed descent set carries a natural representation of the group, called a descent representation. Descent representations of Weyl groups were first introduced by Solomon [30] as alternating sums of permutation representations. This concept was extended to arbitrary Coxeter groups, using a different construction, by Kazhdan and Lusztig [22, [21, §7.15]. For Weyl groups of type $A$, these representations also appear in the top homology of certain (Cohen-Macaulay) rank-selected posets 34. Another description (for type $A)$ is by means of zig-zag diagrams [19, 16 .

In this paper we give a new construction of descent representations for Weyl groups of type $A$, using the coinvariant algebra as a representation space. This viewpoint gives rise to a new extension for type $B$, which refines the one by Solomon.

The construction of a basis for the coinvariant algebra is important for many applications, and has been approached from different viewpoints. A geometric approach identifies the coinvariant algebra with the cohomology ring $H^{*}(G / B)$ of

Received by the editors October 13, 2002 and, in revised form, August 15, 2003.

2000 Mathematics Subject Classification. Primary 05E10, 13A50; Secondary 05A19, 13F20, $20 \mathrm{C} 30$.

The research of all authors was supported in part by the EC's IHRP programme, within the Research Training Network "Algebraic Combinatorics in Europe", grant HPRN-CT-2001-00272, by the Israel Science Foundation, founded by the Israel Academy of Sciences and Humanities, and by internal research grants from Bar-Ilan University. 
the flag variety. This leads to the Schubert basis [7, 10], and applies to any Weyl group. This identification also appears in Springer's construction of irreducible representations [31]; see also [15]. Barcelo [6] found bases for the resulting quotients. An algebraic approach, applying Young symmetrizers, was used by Ariki, Terasoma and Yamada [39, 5] to produce a basis compatible with the decomposition into irreducible representations. This was extended to complex reflection groups in [26].

A combinatorial approach, which produces a basis of monomials, was presented by Garsia and Stanton in [17] (see also [13, 37]). They actually presented a basis for a finite-dimensional quotient of the Stanley-Reisner ring arising from a finite Weyl group. For type $A$, unlike other types, this quotient is isomorphic to the coinvariant algebra. The Garsia-Stanton descent basis for type $A$ may be constructed from the coinvariant algebra via a straightening algorithm [4]. Using a reformulation of this algorithm we give a natural construction of Solomon's descent representations as factors of the coinvariant algebra of type $A$.

An analogue of the descent basis for type $B$ is now given. This analogue (again consisting of monomials) involves extended descent sets and new combinatorial statistics. An extension of the construction of descent representations, using the new basis for type $B$, gives rise to a family of descent representations, refining Solomon's. A decomposition of these descent representations into irreducibles, refining theorems of Lusztig and Stanley [33, Prop. 4.11], 28, Theorem 8.8] (for type $A$ ) and Stembridge 38] (for type $B$ ), is carried out using a multivariate version of Stanley's formula for the principal specialization of Schur functions.

This algebraic setting is then applied to obtain new multivariate combinatorial identities. Suitable Hilbert series are computed and compared to each other and to generating functions of multivariate statistics. The resulting identities present a far reaching generalization of bivariate identities from [18], [14], and [1].

1.2. Main results. Let $W$ be a classical Weyl group of type $A$ or $B$, and let $I_{n}^{W}$ be the ideal of the polynomial ring $P_{n}:=\mathbf{Q}\left[x_{1}, \ldots, x_{n}\right]$ generated by $W$-invariant polynomials without a constant term. The quotient $P_{n} / I_{n}^{W}$ is called the coinvariant algebra of $W$. See Subsection 2.5 below.

For any partition $\lambda$ with (at most) $n$ parts, let $P_{\lambda}^{\unlhd}$ be the subspace of the polynomial ring $P_{n}=\mathbf{Q}\left[x_{1}, \ldots, x_{n}\right]$ spanned by all monomials whose exponent partition is dominated by $\lambda$, and let $R_{\lambda}$ be a distinguished quotient of the image of $P_{\lambda}^{\unlhd}$ under the projection of $P_{n}$ onto the coinvariant algebra. For precise definitions see Subsections 3.5 and 5.3. We will show that the homogeneous components of the coinvariant algebra decompose as direct sums of certain $R_{\lambda}$ 's. This will be done using an explicit construction of a basis for $R_{\lambda}$. The construction of this basis involves new statistics on $S_{n}$ and $B_{n}$.

1.2.1. New statistics. Let $\Sigma$ be a linearly ordered alphabet. For any finite sequence $\sigma=\left(\sigma_{1}, \ldots, \sigma_{n}\right)$ of letters in $\Sigma$ define

$$
\operatorname{Des}(\sigma):=\left\{i \mid \sigma_{i}>\sigma_{i+1}\right\},
$$

the descent set of $\sigma$, and

$$
d_{i}(\sigma):=|\{j \in \operatorname{Des}(\sigma): j \geq i\}|,
$$

the number of descents in $\sigma$ from position $i$ on. 
If $\Sigma$ consists of integers, let

$$
\begin{gathered}
N e g(\sigma):=\left\{i \mid \sigma_{i}<0\right\} ; \\
n_{i}(\sigma):=|\{j \in N e g(\sigma): j \geq i\}| ; \\
\varepsilon_{i}(\sigma):= \begin{cases}1, & \text { if } \sigma_{i}<0, \\
0, & \text { otherwise }\end{cases}
\end{gathered}
$$

and

$$
f_{i}(\sigma):=2 d_{i}(\sigma)+\varepsilon_{i}(\sigma) .
$$

The statistics $f_{i}(\sigma)$ refine the flag-major index $f m a j(\sigma)$, which was introduced and studied in 2, 3, 1].

For various properties of these statistics see Sections 3, 5, and 6 below.

1.2.2. The Garsia-Stanton descent basis and its extension. Garsia and Stanton [17. associated the monomial

$$
a_{\pi}:=\prod_{i \in \operatorname{Des}(\pi)}\left(x_{\pi(1)} \cdots x_{\pi(i)}\right)
$$

to any $\pi \in S_{n}$. It should be noted that in our notation $a_{\pi}=\prod_{i=1}^{n} x_{\pi(i)}^{d_{i}(\pi)}$. Using Stanley-Reisner rings, Garsia and Stanton [17. showed that the set $\left\{a_{\pi}+I_{n} \mid \pi \in\right.$ $\left.S_{n}\right\}$ forms a basis for the coinvariant algebra of type $A$. This basis will be called the descent basis. The Garsia-Stanton approach is not applicable to the coinvariant algebras of other Weyl groups. In this paper we extend the descent basis to the Weyl groups of type $B$.

We associate the monomial

$$
b_{\sigma}:=\prod_{i=1}^{n} x_{|\sigma(i)|}^{f_{i}(\sigma)}
$$

to any $\sigma \in B_{n}$.

Theorem 1.1 (See Corollary 5.3). The set

$$
\left\{b_{\sigma}+I_{n}^{B} \mid \sigma \in B_{n}\right\}
$$

forms a basis for the coinvariant algebra of type $B$.

1.2.3. Descent representations. For a monomial $m$ in the polynomial ring $P_{n}=$ $\mathbf{Q}\left[x_{1}, \ldots, x_{n}\right]$, let the exponent partition $\lambda(m)$ be the partition obtained by rearranging the exponents in a weakly decreasing order. For any partition $\lambda$ with at most $n$ parts, let $P_{\lambda}^{\triangleleft}$ be the subspace of $P_{n}$ spanned by all monomials whose exponent partition is dominated by $\lambda$ :

$$
P_{\lambda}^{\unlhd}:=\operatorname{span}_{\mathbf{Q}}\{m \mid \lambda(m) \unlhd \lambda\} .
$$

Similarly, define $P_{\lambda}^{\triangleleft}$ by strict dominance:

$$
P_{\lambda}^{\triangleleft}:=\operatorname{span}_{\mathbf{Q}}\{m \mid \lambda(m) \triangleleft \lambda\} .
$$

Now consider the canonical projection of $P_{n}$ onto the coinvariant algebra

$$
\psi: P_{n} \longrightarrow P_{n} / I_{n}
$$

Define $R_{\lambda}$ to be a quotient of images under this map:

$$
R_{\lambda}:=\psi\left(P_{\lambda}^{\triangleleft}\right) / \psi\left(P_{\lambda}^{\triangleleft}\right) .
$$

Then $R_{\lambda}$ is an $S_{n}$-module. 
For any subset $S \subseteq\{1, \ldots, n\}$ define a partition

$$
\lambda_{S}:=\left(\lambda_{1}, \ldots, \lambda_{n}\right)
$$

by

$$
\lambda_{i}:=|S \cap\{i, \ldots, n\}| .
$$

Using a straightening algorithm for the descent basis it is shown that $R_{\lambda} \neq 0$ if and only if $\lambda=\lambda_{S}$ for some $S \subseteq[n-1]$ (Corollary 3.10), and that a basis for $R_{\lambda_{S}}$ may be indexed by the permutations with descent set equal to $S$ (Corollary 3.11). Let $R_{k}$ be the $k$-th homogeneous component of the coinvariant algebra $P_{n} / I_{n}$.

Theorem 1.2 (See Theorem[3.12). For every $0 \leq k \leq\left(\begin{array}{l}n \\ 2\end{array}\right)$,

$$
R_{k} \cong \bigoplus_{S} R_{\lambda_{S}}
$$

as $S_{n}$-modules, where the sum is over all subsets $S \subseteq[n-1]$ such that $\sum_{i \in S} i=k$.

Let

$$
R_{\lambda}^{B}:=\psi^{B}\left(P_{\lambda}^{\unlhd}\right) / \psi^{B}\left(P_{\lambda}^{\triangleleft}\right),
$$

where $\psi^{B}: P_{n} \longrightarrow P_{n} / I_{n}^{B}$ is the canonical map from $P_{n}$ onto the coinvariant algebra of type $B$. For subsets $S_{1} \subseteq[n-1]$ and $S_{2} \subseteq[n]$, let $\lambda_{S_{1}, S_{2}}$ be the vector

$$
\lambda_{S_{1}, S_{2}}:=2 \lambda_{S_{1}}+\mathbf{1}_{S_{2}},
$$

where $\lambda_{S_{1}}$ is as above and $\mathbf{1}_{S_{2}} \in\{0,1\}^{n}$ is the characteristic vector of $S_{2}$. Again, $R_{\lambda}^{B} \neq 0$ if and only if $\lambda=\lambda_{S_{1}, S_{2}}$ for some $S_{1} \subseteq[n-1], S_{2} \subseteq[n]$ (Corollary [5.6). In this case, a basis for $R_{\lambda_{S_{1}, S_{2}}}^{B}$ may be indexed by the signed permutations $\sigma \in B_{n}$ with $\operatorname{Des}(\sigma)=S_{1}$ and $\operatorname{Neg}(\sigma)=S_{2}$ (Corollary 5.7).

Let $R_{k}^{B}$ be the $k$-th homogeneous component of the coinvariant algebra of type $B$. The following theorem is a $B$-analogue of Theorem 1.2

Theorem 1.3 (See Theorem 5.8). For every $0 \leq k \leq n^{2}$,

$$
R_{k}^{B} \cong \bigoplus_{S_{1}, S_{2}} R_{\lambda_{S_{1}, S_{2}}}^{B}
$$

as $B_{n}$-modules, where the sum is over all subsets $S_{1} \subseteq[n-1]$ and $S_{2} \subseteq[n]$ such that $\lambda_{S_{1}, S_{2}}$ is a partition and

$$
2 \cdot \sum_{i \in S_{1}} i+\left|S_{2}\right|=k .
$$

\subsubsection{Decomposition into irreducibles.}

Theorem 1.4 (See Theorem 4.1). For any subset $S \subseteq[n-1]$ and partition $\mu \vdash n$, the multiplicity in $R_{\lambda_{S}}$ of the irreducible $S_{n}$-representation corresponding to $\mu$ is

$$
m_{S, \mu}:=|\{T \in S Y T(\mu) \mid \operatorname{Des}(T)=S\}|,
$$

the number of standard Young tableaux of shape $\mu$ and descent set $S$.

This theorem refines the well-known decomposition (due, independently, to Lusztig and Stanley) of each homogeneous component of the coinvariant algebra into irreducibles. See Subsection 2.5. 
For type $B$ we have

Theorem 1.5. For any pair of subsets $S_{1} \subseteq[n-1], S_{2} \subseteq[n]$, and a bipartition $\left(\mu^{1}, \mu^{2}\right)$ of $n$, the multiplicity of the irreducible $B_{n}$-representation corresponding to $\left(\mu^{1}, \mu^{2}\right)$ in $R_{\lambda_{S_{1}, S_{2}}}^{B}$ is

$$
m_{S_{1}, S_{2}, \mu^{1}, \mu^{2}}:=\mid\left\{T \in S Y T\left(\mu^{1}, \mu^{2}\right) \mid \operatorname{Des}(T)=S_{1} \text { and } \operatorname{Neg}(T)=S_{2}\right\} \mid,
$$

the number of pairs of standard Young tableaux of shapes $\mu^{1}$ and $\mu^{2}$ with descent set $S_{1}$ and sets of entries $[n] \backslash S_{2}$ and $S_{2}$, respectively.

For definitions and more details see Subsection 5.4 and Theorem 5.9 below.

The proofs apply multivariate extensions of Stanley's formula for the principal specialization of a Schur function. See Lemmas 4.3 and 5.10 below.

1.2.5. Combinatorial identities. For any partition $\lambda=\left(\lambda_{1}, \ldots, \lambda_{n}\right)$ with at most $n$ parts define

$$
m_{j}(\lambda):=\left|\left\{1 \leq i \leq n \mid \lambda_{i}=j\right\}\right| \quad(\forall j \geq 0) .
$$

By considering Hilbert series of the polynomial ring with respect to rearranged multi-degree and applying the Straightening Lemma for the coinvariant algebra of type $A$ we obtain

Theorem 1.6 (See Theorem 6.2). For any positive integer $n$

$$
\sum_{\ell(\lambda) \leq n}\left(\begin{array}{c}
n \\
m_{0}(\lambda), m_{1}(\lambda), \ldots
\end{array}\right) \prod_{i=1}^{n} q_{i}^{\lambda_{i}}=\frac{\sum_{\pi \in S_{n}} \prod_{i=1}^{n} q_{i}^{d_{i}(\pi)}}{\prod_{i=1}^{n}\left(1-q_{1} \cdots q_{i}\right)}
$$

in $\boldsymbol{Z}\left[\left[q_{1}, \ldots, q_{n}\right]\right]$, where the sum on the left-hand side is taken over all partitions with at most $n$ parts.

This theorem generalizes Gessel's theorem for the bivariate distribution of descent number and major index 18 .

The main combinatorial result for type $B$ asserts:

Theorem 1.7 (See Theorem6.1). For any positive integer $n$

$$
\sum_{\sigma \in B_{n}} \prod_{i=1}^{n} q_{i}^{d_{i}(\sigma)+n_{i}\left(\sigma^{-1}\right)}=\sum_{\sigma \in B_{n}} \prod_{i=1}^{n} q_{i}^{2 d_{i}(\sigma)+\varepsilon_{i}(\sigma)} .
$$

For further identities see Section 6. In particular, it is shown that central results from [1] follow from Theorem 1.7.

\section{Preliminaries}

2.1. Notation. Let $\boldsymbol{P}:=\{1,2,3, \ldots\}, \boldsymbol{N}:=\boldsymbol{P} \cup\{0\}, \boldsymbol{Z}$ be the ring of integers, and $\mathbf{Q}$ be the field of rational numbers; for $a \in \boldsymbol{N}$ let $[a]:=\{1,2, \ldots, a\}$ (where $[0]:=\emptyset)$. Given $n, m \in \boldsymbol{Z}, n \leq m$, let $[n, m]:=\{n, n+1, \ldots, m\}$. For $S \subset \boldsymbol{N}$ write $S=\left\{a_{1}, \ldots, a_{r}\right\}<$ to mean that $S=\left\{a_{1}, \ldots, a_{r}\right\}$ and $a_{1}<\ldots<a_{r}$. The cardinality of a set $A$ will be denoted by $|A|$. More generally, given a multiset $M=\left\{1^{a_{1}}, 2^{a_{2}}, \ldots, r^{a_{r}}\right\}$ denote by $|M|$ its cardinality, so $|M|=\sum_{i=1}^{r} a_{i}$.

Given a variable $q$ and a commutative ring $R$, denote by $R[q]$ (respectively, $R[[q]]$ ) the ring of polynomials (respectively, formal power series) in $q$ with coefficients in $R$. For $i \in \boldsymbol{N}$ let, as customary, $[i]_{q}:=1+q+q^{2}+\ldots+q^{i-1}$ (so $[0]_{q}=0$ ). Given a vector $v=\left(v_{1}, \ldots, v_{n}\right) \in \boldsymbol{P}^{n}$ and a sequence of variables $x_{1}, \ldots, x_{n}$ denote by $\bar{x}^{v}$ the monomial $\prod_{i=1}^{n} x_{i}^{v_{i}} \in R\left[x_{1}, \ldots, x_{n}\right]$. 
2.2. Sequences and permutations. Let $\Sigma$ be a linearly ordered alphabet. Given a sequence $\sigma=\left(\sigma_{1}, \ldots, \sigma_{n}\right) \in \Sigma^{n}$ we say that a pair $(i, j) \in[n] \times[n]$ is an inversion of $\sigma$ if $i<j$ and $\sigma_{i}>\sigma_{j}$. We say that $i \in[n-1]$ is a descent of $\sigma$ if $\sigma_{i}>\sigma_{i+1}$;

$$
\operatorname{Des}(\sigma):=\left\{1 \leq i \leq n-1 \mid \sigma_{i}>\sigma_{i+1}\right\}
$$

is the descent set of $\sigma$. Denote by $\operatorname{inv}(\sigma)$ (respectively, des $(\sigma)$ ) the number of inversions (respectively, descents) of $\sigma$. We also let

$$
\operatorname{maj}(\sigma):=\sum_{i \in \operatorname{Des}(\sigma)} i
$$

and call it the major index of $\sigma$.

Given a set $T$ let $S(T)$ be the set of all bijections $\pi: T \rightarrow T$, and $S_{n}:=S([n])$. For $\pi \in S_{n}$ write $\pi=\pi_{1} \ldots \pi_{n}$ to mean that $\pi(i)=\pi_{i}$, for $i=1, \ldots, n$. Any $\pi \in S_{n}$ may also be written in disjoint cycle form (see, e.g., [35, p. 17]), usually omitting the 1-cycles of $\pi$. For example, $\pi=365492187$ may also be written as $\pi=(9,7,1,3,5)(2,6)$. Given $\pi, \tau \in S_{n}$ let $\pi \tau:=\pi \circ \tau$ (composition of functions) so that, for example, $(1,2)(2,3)=(1,2,3)$.

Denote by $B_{n}$ the group of all bijections $\sigma$ of the set $[-n, n] \backslash\{0\}$ onto itself such that

$$
\sigma(-a)=-\sigma(a)
$$

for all $a \in[-n, n] \backslash\{0\}$, with composition as the group operation. This group is usually known as the group of "signed permutations" on $[n]$, or as the hyperoctahedral group of rank $n$. We identify $S_{n}$ as a subgroup of $B_{n}$, and $B_{n}$ as a subgroup of $S_{2 n}$, in the natural ways.

For $\sigma \in B_{n}$ write $\sigma=\left[a_{1}, \ldots, a_{n}\right]$ to mean that $\sigma(i)=a_{i}$ for $i=1, \ldots, n$, and (using the natural linear order on $[-n, n] \backslash\{0\}$ ) let

$$
\begin{array}{ll}
\operatorname{inv}(\sigma):=\operatorname{inv}\left(a_{1}, \ldots, a_{n}\right), & \operatorname{des}(\sigma):=\operatorname{des}\left(a_{1}, \ldots, a_{n}\right), \\
\operatorname{Neg}(\sigma):=\left\{i \in[n]: a_{i}<0\right\}, & \operatorname{neg}(\sigma):=|\operatorname{Neg}(\sigma)|, \\
\operatorname{maj}(\sigma):=\operatorname{maj}\left(a_{1}, \ldots, a_{n}\right), & \operatorname{fmaj}(\sigma):=2 \cdot \operatorname{maj}(\sigma)+\operatorname{neg}(\sigma) .
\end{array}
$$

The statistic fmaj was introduced in [2, 3] and further studied in [1].

2.3. Partitions and tableaux. Let $n$ be a nonnegative integer. A partition of $n$ is an infinite sequence of nonnegative integers with finitely many nonzero terms $\lambda=\left(\lambda_{1}, \lambda_{2}, \ldots\right)$, where $\lambda_{1} \geq \lambda_{2} \geq \ldots$ and $\sum_{i=1}^{\infty} \lambda_{i}=n$. The sum $\sum \lambda_{i}=n$ is called the size of $\lambda$, denoted $|\lambda|$; also write $\lambda \vdash n$. The number of parts of $\lambda, \ell(\lambda)$, is the maximal $j$ for which $\lambda_{j}>0$. The unique partition of $n=0$ is the empty partition $\emptyset=(0,0, \ldots)$, which has length $\ell(\emptyset):=0$. For a partition $\lambda=\left(\lambda_{1}, \ldots, \lambda_{k}, \ldots\right)$ define the conjugate partition $\lambda^{\prime}=\left(\lambda_{1}^{\prime}, \ldots, \lambda_{i}^{\prime}, \ldots\right)$ by letting $\lambda_{i}^{\prime}$ be the number of parts of $\lambda$ that are $\geq i(\forall i \geq 1)$.

The dominance partial order on partitions is defined as follows: For any two partitions $\mu$ and $\lambda$ of the same integer, $\mu$ dominates $\lambda$ (denoted $\mu \unrhd \lambda$ ) if and only if $\sum_{j=1}^{i} \mu_{j} \geq \sum_{j=1}^{i} \lambda_{j}$ for all $i$ (and, by assumption, $\sum_{j=1}^{\infty} \mu_{j}=\sum_{j=1}^{\infty} \lambda_{j}$ ).

The subset $\left\{(i, j) \mid i, j \in \mathbf{P}, j \leq \lambda_{i}\right\}$ of $\mathbf{P}^{2}$ is called the Young diagram of shape $\lambda$. $(i, j)$ is the cell in row $i$ and column $j$. The diagram of the conjugate shape $\lambda^{\prime}$ may be obtained from the diagram of shape $\lambda$ by interchanging rows and columns.

A Young tableau of shape $\lambda$ is obtained by inserting the integers $1, \ldots, n$ (where $n=|\lambda|)$ as entries in the cells of the Young diagram of shape $\lambda$, allowing no 
repetitions. A standard Young tableau of shape $\lambda$ is a Young tableau whose entries increase along rows and columns.

We shall draw Young tableaux as in the following example.

\section{Example.}

$\begin{array}{ccccc}1 & 3 & 4 & 6 & 9 \\ 2 & 7 & 8 & 11 & \\ 5 & 10 & & & \end{array}$

A descent in a standard Young tableau $T$ is an entry $i$ such that $i+1$ is strictly south (and hence weakly west) of $i$. Denote the set of all descents in $T$ by $\operatorname{Des}(T)$. The descent number and the major index (for tableaux) are defined as follows:

$$
\operatorname{des}(T):=\sum_{i \in \operatorname{Des}(T)} 1 ; \quad \operatorname{maj}(T):=\sum_{i \in \operatorname{Des}(T)} i .
$$

Example. Let $T$ be the standard Young tableau drawn above. Then $\operatorname{Des}(T)=$ $\{1,4,6,9\}, \operatorname{des}(T)=4$, and $\operatorname{maj}(T)=1+4+6+9=20$.

A semistandard Young tableau of shape $\lambda$ is obtained by inserting positive integers as entries in the cells of the Young diagram of shape $\lambda$, so that the entries weakly increase along rows and strictly increase down columns. A reverse semistandard Young tableau is obtained by inserting positive integers into the diagram so that the entries weakly decrease along rows and strictly decrease down columns.

A bipartition of $n$ is a pair $\left(\lambda^{1}, \lambda^{2}\right)$ of partitions of total size $\left|\lambda^{1}\right|+\left|\lambda^{2}\right|=n$. A (skew) diagram of shape $\left(\lambda^{1}, \lambda^{2}\right)$ is the disjoint union of a diagram of shape $\lambda^{1}$ and a diagram of shape $\lambda^{2}$, where the second diagram lies southwest of the first. A standard Young tableau $T=\left(T^{1}, T^{2}\right)$ of shape $\left(\lambda^{1}, \lambda^{2}\right)$ is obtained by inserting the integers $1,2, \ldots, n$ as entries in the cells, such that the entries increase along rows and columns. The descent set $\operatorname{Des}(T)$, the descent number $\operatorname{des}(T)$, and the major index $\operatorname{maj}(T)$ of $T$ are defined as above. The negative set, $N e g(T)$, of such a tableau $T$ is the set of entries in the cells of $\lambda^{2}$. Define $\operatorname{neg}(T):=|\operatorname{Neg}(T)|$ and

$$
\operatorname{fmaj}(T):=2 \cdot \operatorname{maj}(T)+\operatorname{neg}(T) .
$$

Example. Let $T$ be

$$
\begin{array}{lllll} 
& & 2 & 5 & 6 \\
& & 3 & & \\
1 & 7 & & & \\
4 & 8 & & &
\end{array}
$$

We shall also consider $T$ as a pair of tableaux

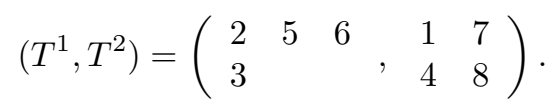

$T$ is a standard Young tableau of shape $((3,1),(2,2)) ; \operatorname{Des}(T)=\{2,3,6,7\}, \operatorname{des}(T)$ $=4, \operatorname{maj}(T)=18, \operatorname{Neg}(T)=\{1,4,7,8\}, \operatorname{neg}(T)=4$, and $\operatorname{fmaj}(T)=40$.

Denote by $S Y T(\lambda)$ the set of all standard Young tableaux of shape $\lambda$. Similarly, $\operatorname{SSYT}(\lambda)$ and $\operatorname{RSSYT}(\lambda)$ denote the sets of all semistandard and reverse semistandard Young tableaux of shape $\lambda$, respectively. $S Y T\left(\lambda^{1}, \lambda^{2}\right)$ denotes the set of all standard Young tableaux of shape $\left(\lambda^{1}, \lambda^{2}\right)$. 
Let $\hat{T}$ be a (reverse) semistandard Young tableau. The content vector $\operatorname{cont}(\hat{T})=$ $\left(m_{1}, m_{2}, \ldots\right)$ is defined by

$$
m_{i}:=\mid\{\text { cells in } \hat{T} \text { with entry } i\} \mid \quad(\forall i \geq 1) .
$$

2.4. Symmetric functions. Let $\Lambda\left(\Lambda_{n}\right)$ be the ring of symmetric functions in infinitely many (resp. $n$ ) variables. In this paper we consider three bases for the vector space $\Lambda$ :

The elementary symmetric functions are defined as follows. For a nonempty partition $\lambda=\left(\lambda_{1}, \lambda_{2}, \ldots, \lambda_{t}\right)$

$$
e_{\lambda}(\bar{x}):=\prod_{i=1}^{t} e_{\lambda_{i}}(\bar{x})
$$

where $\bar{x}=\left(x_{1}, x_{2}, \ldots\right)$ and for any $k \in \boldsymbol{P}$

$$
e_{k}(\bar{x}):=\sum_{i_{1}<i_{2}<\cdots<i_{k}} x_{i_{1}} \cdots x_{i_{k}} .
$$

The power sum symmetric functions are defined as follows. For a nonempty partition $\lambda=\left(\lambda_{1}, \lambda_{2}, \ldots, \lambda_{t}\right)$

$$
p_{\lambda}(\bar{x}):=\prod_{i=1}^{t} p_{\lambda_{i}}(\bar{x})
$$

where for any $k \in \boldsymbol{P}$

$$
p_{k}(\bar{x}):=\sum_{i=1}^{\infty} x_{i}^{k} .
$$

The Schur functions are defined in a different manner. For a nonempty partition $\lambda$ define

$$
s_{\lambda}(\bar{x}):=\sum_{T \in S S Y T(\lambda)} \bar{x}^{\operatorname{cont}(T)} .
$$

For the empty partition $\lambda=\emptyset$, define $e_{\emptyset}(\bar{x})=p_{\emptyset}(\bar{x})=s_{\emptyset}(\bar{x}):=1$.

Claim 2.1 (See [36, Prop. 7.10.4]).

$$
s_{\lambda}(\bar{x})=\sum_{T \in R S S Y T(\lambda)} \bar{x}^{\operatorname{cont}(T)} .
$$

Note that corresponding spanning sets for $\Lambda_{n}$ are obtained by substituting $x_{n+1}=x_{n+2}=\cdots=0$.

The conjugacy classes of the symmetric group $S_{n}$ are described by their cycle type; thus, by partitions of $n$. The irreducible representations of $S_{n}$ are also indexed by these partitions (cf. [29]).

Let $\lambda$ and $\mu$ be partitions of $n$. Denote by $\chi_{\mu}^{\lambda}$ the value, at a conjugacy class of cycle type $\mu$, of the character of the irreducible $S_{n}$-representation corresponding to $\lambda$. These character values are entries in the transition matrix between two of the above bases. This fact was discovered and applied by Frobenius as an efficient tool for calculating characters (cf. [36, p. 401]).

Frobenius formula ([36, Corollary 7.17.4]). For any partition $\mu$ of $n$

$$
p_{\mu}(\bar{x})=\sum_{\lambda \vdash n} \chi_{\mu}^{\lambda} s_{\lambda}(\bar{x}),
$$

where the sum runs through all partitions $\lambda$ of $n$. 
Recall that the Weyl group $B_{n}$ may be described as the group of all signed permutations of order $n$ (Subsection 2.2). The conjugacy classes of $B_{n}$ are described by the signed cycle type (i.e., each cycle is described by its length and the product of the signs of its entries). Thus, the conjugacy classes are described by ordered pairs of partitions whose total size is $n$ : the first partition consists of the lengths of the positive cycles, while the second consists of the lengths of the negative cycles. The irreducible representations of $B_{n}$ are also indexed by these pairs of partitions; cf. 24, §I, Appendix B].

For two partitions $\mu^{1}$ and $\mu^{2}$, and two infinite sets of independent variables $\bar{x}=x_{1}, x_{2}, \ldots$ and $\bar{y}=y_{1}, y_{2}, \ldots$, define

$$
p_{\mu^{1}, \mu^{2}}(\bar{x}, \bar{y}):=\prod_{i}\left(p_{\mu_{i}^{1}}(\bar{x})+p_{\mu_{i}^{1}}(\bar{y})\right) \cdot \prod_{j}\left(p_{\mu_{j}^{2}}(\bar{x})-p_{\mu_{j}^{2}}(\bar{y})\right) .
$$

Let $\chi_{\mu^{1}, \mu^{2}}^{\lambda^{1}, \lambda^{2}}$ be the irreducible $B_{n^{-}}$character indexed by $\left(\lambda^{1}, \lambda^{2}\right)$ evaluated at a conjugacy class of cycle type $\left(\mu^{1}, \mu^{2}\right)$. Then

Frobenius formula for $B_{n}$ ([24, p. 178]). With the above notations, for any bipartition $\left(\mu^{1}, \mu^{2}\right)$ of $n$

$$
p_{\mu^{1}, \mu^{2}}(\bar{x}, \bar{y})=\sum_{\lambda^{1}, \lambda^{2}} \chi_{\mu^{1}, \mu^{2}}^{\lambda^{1}, \lambda^{2}} s_{\lambda^{1}}(\bar{x}) s_{\lambda^{2}}(\bar{y}),
$$

where the sum runs through all ordered pairs of partitions $\left(\lambda^{1}, \lambda^{2}\right)$ of total size $\left|\lambda^{1}\right|+\left|\lambda^{2}\right|=n$.

2.5. The coinvariant algebra. The groups $S_{n}$ and $B_{n}$ have natural actions on the ring of polynomials $P_{n}$ (cf. [21 $\left.\S 3.1\right]$ ). $S_{n}$ acts by permuting the variables, and $B_{n}$ acts by permuting the variables and multiplying by \pm 1 . The ring of $S_{n}$-invariant polynomials is $\Lambda_{n}$, the ring of symmetric functions in $x_{1}, \ldots, x_{n}$. Similarly, the ring of $B_{n}$-invariant polynomials is $\Lambda_{n}^{B}$, the ring of symmetric functions in $x_{1}^{2}, \ldots, x_{n}^{2}$. Let $I_{n}, I_{n}^{B}$ be the ideals of $P_{n}$ generated by the elements of $\Lambda_{n}, \Lambda_{n}^{B}$ (respectively) without constant term. The quotient $P_{n} / I_{n}\left(P_{n} / I_{n}^{B}\right)$ is called the coinvariant algebra of $S_{n}\left(B_{n}\right)$. Each group acts naturally on its coinvariant algebra. The resulting representation is isomorphic to the regular representation. See, e.g., [21] $\S 3.6]$ and [20, $\S I I .3]$.

Let $R_{k}\left(0 \leq k \leq\left(\begin{array}{c}n \\ 2\end{array}\right)\right)$ be the $k$-th homogeneous component of the coinvariant algebra of $S_{n}: P_{n} / I_{n}=\bigoplus_{k} R_{k}$. Each $R_{k}$ is an $S_{n}$-module. The following theorem is apparently due to G. Lusztig (unpublished) and, independently, to R. Stanley [33. Prop. 4.11]. It was also proved by Kraskiewicz and Weyman [23]; see [28, p. 215].

Lusztig-Stanley theorem ([33, Prop. 4.11], [28, Theorem 8.8]). For any $0 \leq$ $k \leq\left(\begin{array}{l}n \\ 2\end{array}\right)$ and $\mu \vdash n$, the multiplicity in $R_{k}$ of the irreducible $S_{n}$-representation corresponding to $\mu$ is

$$
m_{k, \mu}=|\{T \in S Y T(\mu) \mid \operatorname{maj}(T)=k\}| .
$$

The following $B$-analogue (in different terminology) was proved in 38 . Here $R_{k}^{B}$ is the $k$-th homogeneous component of the coinvariant algebra of $B_{n}$.

Stembridge's theorem. For any $0 \leq k \leq n^{2}$ and bipartition $\left(\mu^{1}, \mu^{2}\right)$ of $n$, the multiplicity in $R_{k}^{B}$ of the irreducible $B_{n}$-representation corresponding to $\left(\mu^{1}, \mu^{2}\right)$ is

$$
m_{k, \mu^{1}, \mu^{2}}=\left|\left\{T \in S Y T\left(\mu^{1}, \mu^{2}\right) \mid \operatorname{fmaj}(T)=k\right\}\right| .
$$


2.6. The Garsia-Stanton descent basis (Type $A$ ). For any $\pi \in S_{n}$ define the monomial

$$
a_{\pi}:=\prod_{j \in \operatorname{Des}(\pi)}\left(x_{\pi(1)} \cdots x_{\pi(j)}\right) .
$$

Using Stanley-Reisner rings Garsia and Stanton showed that the set $\left\{a_{\pi}+I_{n} \mid \pi \in\right.$ $\left.S_{n}\right\}$ forms a basis for the coinvariant algebra of type $A$ [17]. This basis will be called the descent basis. Unfortunately, this approach does not give a basis for the coinvariant algebras of other Weyl groups. In this paper we shall find an analogue of the descent basis for $B_{n}$. Our approach will allow us to obtain refinements of the Lusztig-Stanley and Stembridge theorems.

\section{Construction of Descent Representations}

In this section we introduce several combinatorial concepts (Subsections 3.1 and 3.2). Then we present a straightening algorithm for the expansion of an arbitrary monomial in $P_{n}$ in terms of descent basis elements, with coefficients from $\Lambda_{n}$ (Subsections 3.3 and 3.4). This algorithm is essentially equivalent to the one presented by Allen [4, but our formulation leads naturally to descent representations and may be extended to type $B$ (Subsections 3.5 and 5.2).

3.1. Descent statistics. In this subsection we introduce a family of descent statistics for sequences of letters from a linearly ordered alphabet.

Let $\Sigma$ be a linearly ordered alphabet. For any sequence $\sigma$ of $n$ letters from $\Sigma$ define

$$
d_{i}(\sigma):=|\{j \in \operatorname{Des}(\sigma): j \geq i\}| \quad(1 \leq i \leq n),
$$

the number of descents in $\sigma$ from position $i$ on. Clearly,

$$
d_{1}(\sigma)=\operatorname{des}(\sigma)
$$

and

$$
\sum_{i=1}^{n} d_{i}(\sigma)=\operatorname{maj}(\sigma)
$$

Note that $d_{n}(\sigma)=0$.

Also, for any sequence $\sigma$ from $\Sigma$

$$
\begin{gathered}
d_{i}(\sigma) \geq d_{i+1}(\sigma), \quad 1 \leq i<n, \\
d_{i}(\sigma)=d_{i+1}(\sigma) \Longrightarrow \sigma(i)<\sigma(i+1), \\
d_{i}(\sigma)>d_{i+1}(\sigma) \Longrightarrow \sigma(i)>\sigma(i+1) \text { and } d_{i}(\sigma)=d_{i+1}(\sigma)+1 .
\end{gathered}
$$

It follows from (3.2) and (3.3) that the sequence $\left(d_{1}(\sigma), d_{2}(\sigma), \ldots, d_{n}(\sigma)\right)$ is a partition of $\operatorname{maj}(\sigma)$. It follows from (3.4) and (3.5) that this sequence uniquely determines the descent set of $\sigma$. More explicitly, the partition conjugate to $\left(d_{1}(\sigma), \ldots\right.$, $\left.d_{n}(\sigma)\right)$ is obtained by writing the elements of $\operatorname{Des}(\sigma)$ in decreasing order.

Finally, for a permutation $\pi \in S_{n}$, let $a_{\pi}$ be the corresponding descent basis element. Then, clearly,

$$
a_{\pi}=\prod_{i=1}^{n} x_{\pi(i)}^{d_{i}(\pi)}
$$


3.2. The index permutation and the exponent partition. The index permutation of a monomial $m=\prod_{i=1}^{n} x_{i}^{p_{i}} \in P_{n}$ is the unique permutation $\pi=\pi(m) \in S_{n}$ such that

$$
p_{\pi(i)} \geq p_{\pi(i+1)} \quad(1 \leq i<n)
$$

and

$$
p_{\pi(i)}=p_{\pi(i+1)} \Longrightarrow \pi(i)<\pi(i+1) .
$$

In other words, $\pi$ reorders the variables $x_{i}$ by (weakly) decreasing exponents, where the variables with a given exponent are ordered by increasing indices.

Let $m=\prod_{i=1}^{n} x_{i}^{p_{i}}$ be a monomial in $P_{n}, \pi=\pi(m)$ its index permutation, and $a_{\pi}$ the corresponding descent basis element. Then $m=\prod_{i=1}^{n} x_{\pi(i)}^{p_{\pi(i)}}$ and $a_{\pi}=$ $\prod_{i=1}^{n} x_{\pi(i)}^{d_{i}(\pi)}$.

Claim 3.1. The sequence $\left(p_{\pi(i)}-d_{i}(\pi)\right)_{i=1}^{n}$ of exponents in $m / a_{\pi}$ consists of nonnegative integers, and is weakly decreasing:

$$
p_{\pi(i)}-d_{i}(\pi) \geq p_{\pi(i+1)}-d_{i+1}(\pi) \quad(1 \leq i<n) .
$$

Proof. If $\pi(i)<\pi(i+1)$, then $d_{i}(\pi)=d_{i+1}(\pi)$ and the claim follows from $p_{\pi(i)} \geq$ $p_{\pi(i+1)}$. If $\pi(i)>\pi(i+1)$, then $d_{i}(\pi)=d_{i+1}(\pi)+1$ and $p_{\pi(i)}>p_{\pi(i+1)}$, so that $p_{\pi(i)}-p_{\pi(i+1)} \geq 1=d_{i}(\pi)-d_{i+1}(\pi)$. This proves monotonicity. Nonnegativity now follows from $d_{n}(\pi)=0$, implying $p_{\pi(n)}-d_{n}(\pi) \geq 0$.

For a monomial $m=\prod_{i=1}^{n} x_{i}^{p_{i}}$ with index permutation $\pi \in S_{n}$, let

$$
\lambda(m):=\left(p_{\pi(1)}, p_{\pi(2)}, \ldots, p_{\pi(n)}\right)
$$

be its exponent partition. Note that $\lambda(m)$ is a partition of the total degree of $m$.

Define the complementary partition $\mu(m)$ of a monomial $m$ to be the partition conjugate to the partition $\left(p_{\pi(i)}-d_{i}(\pi)\right)_{i=1}^{n}$. Namely,

$$
\mu_{j}:=\left|\left\{i \mid p_{\pi(i)}-d_{i}(\pi) \geq j\right\}\right| \quad(\forall j \geq 1) .
$$

Example. Let $m=x_{1}^{2} x_{2}^{4} x_{3}^{2} x_{5} x_{6}^{3}$ and $n=7$. Then $m=x_{2}^{4} x_{6}^{3} x_{1}^{2} x_{3}^{2} x_{5}^{1} x_{4}^{0} x_{7}^{0}, \lambda(m)=$ $(4,3,2,2,1,0,0), \pi=2613547 \in S_{7},\left(d_{1}(\pi), \ldots, d_{7}(\pi)\right)=(2,2,1,1,1,0,0)$, and $\mu(m)=(4,1)$.

3.3. A partial order for monomials. We shall now define a partial order on the monomials in $P_{n}$.

Comparable monomials will always have the same total degree. Fix such a total degree $p$; we may assume $p \geq 1$.

Definition. For monomials $m_{1}, m_{2}$ of the same total degree $p, m_{1} \prec m_{2}$ if one of the following holds:

(1) $\lambda\left(m_{1}\right) \triangleleft \lambda\left(m_{2}\right)$ (strictly smaller in dominance order); or

(2) $\lambda\left(m_{1}\right)=\lambda\left(m_{2}\right)$ and $\operatorname{inv}\left(\pi\left(m_{1}\right)\right)>\operatorname{inv}\left(\pi\left(m_{2}\right)\right)$.

The partial order " $\prec$ " may be replaced, in this paper, by any linear extension of it; for example, by the linear extension obtained by replacing the dominance order by lexicographic order in (1). (This is common in the definition of Gröbner bases, which partially motivated our definition.) 


\subsection{Straightening.}

Lemma 3.2. Let $m \in P_{n}$ be a monomial and let $1 \leq k \leq n$. Let $S^{(k)}$ be the set of all monomials which appear (with coefficient 1 ) in the expansion of the polynomial $m \cdot e_{k}$, the product of $m$ and the $k$-th elementary symmetric function. Let

$$
m^{(k)}:=m \cdot x_{\pi(1)} \cdot x_{\pi(2)} \cdots x_{\pi(k)},
$$

where $\pi=\pi(m)$ is the index permutation of $m$. Then:

(1) $m^{(k)} \in S^{(k)}$.

(2) $m^{\prime} \in S^{(k)}$ and $m^{\prime} \neq m^{(k)} \Longrightarrow m^{\prime} \prec m^{(k)}$.

Proof. (1) is obvious. For (2), let $m^{\prime} \in S^{(k)}$. Then $m^{\prime}=m \cdot x_{i_{1}} x_{i_{2}} \cdots x_{i_{k}}$, where $1 \leq i_{1}<i_{2}<\cdots<i_{k} \leq n$. Now, let $\lambda_{i}(m)$ be the $i$-th part of the exponent partition of the monomial $m$. Then

$$
\lambda_{i}\left(m^{(k)}\right)-\lambda_{i}(m)= \begin{cases}1, & \text { if } 1 \leq i \leq k \\ 0, & \text { if } i>k,\end{cases}
$$

and also

$$
\begin{gathered}
\lambda_{i}\left(m^{\prime}\right)-\lambda_{i}(m) \in\{0,1\} \quad(\forall i) \\
\sum_{i=1}^{n}\left[\lambda_{i}\left(m^{\prime}\right)-\lambda_{i}(m)\right]=k
\end{gathered}
$$

Thus

$$
\begin{aligned}
& \sum_{i=1}^{t} \lambda_{i}\left(m^{\prime}\right) \leq \sum_{i=1}^{t}\left[\lambda_{i}(m)+1\right]=\sum_{i=1}^{t} \lambda_{i}\left(m^{(k)}\right) \quad(1 \leq t \leq k), \\
& \sum_{i=1}^{t} \lambda_{i}\left(m^{\prime}\right) \leq k+\sum_{i=1}^{t} \lambda_{i}(m)=\sum_{i=1}^{t} \lambda_{i}\left(m^{(k)}\right) \quad(k<t \leq n) .
\end{aligned}
$$

Therefore

$$
\lambda\left(m^{\prime}\right) \unlhd \lambda\left(m^{(k)}\right) .
$$

If $\lambda\left(m^{\prime}\right) \triangleleft \lambda\left(m^{(k)}\right)$, then $m^{\prime} \prec m^{(k)}$, as claimed. Otherwise, $\lambda\left(m^{\prime}\right)=\lambda\left(m^{(k)}\right)$.

Now let

$$
\begin{aligned}
& I_{>}:=\left\{1 \leq i \leq n \mid \lambda_{i}(m)>\lambda_{k}(m)\right\} \\
& I_{=}:=\left\{1 \leq i \leq n \mid \lambda_{i}(m)=\lambda_{k}(m)\right\} \\
& I_{<}:=\left\{1 \leq i \leq n \mid \lambda_{i}(m)<\lambda_{k}(m)\right\}
\end{aligned}
$$

Namely, $i \in I_{>}$if the exponent (in $m$ ) of $x_{\pi(i)}$ is strictly larger than that of $x_{\pi(k)}$; and similarly for $I_{=}$and $I_{<}$. By definition,

$$
\pi\left(m^{(k)}\right)=\pi(m) .
$$

Since $\lambda\left(m^{\prime}\right)=\lambda\left(m^{(k)}\right)$, it follows that $\pi\left(m^{\prime}\right)$ and $\pi(m)$ agree on $I_{<} \cup I_{>}$, but may differ on $I_{=}$. Also, $\pi\left(m^{(k)}\right)=\pi(m)$ is monotone increasing on $I_{=}$. It follows that if $m^{\prime} \neq m^{(k)}$, then $\operatorname{inv}\left(\pi\left(m^{\prime}\right)\right)>\operatorname{inv}\left(\pi\left(m^{(k)}\right)\right)$ and thus $m^{\prime} \prec m^{(k)}$ in the monomial partial order. 
Lemma 3.3. Let $m_{1}$ and $m_{2}$ be monomials in $P_{n}$ of the same total degree, and let $e_{k}$ be the $k$-th elementary symmetric function, $1 \leq k \leq n$. If $m_{1} \prec m_{2}$, then

$$
m_{1}^{(k)} \prec m_{2}^{(k)},
$$

where $m_{i}^{(k)}(i=1,2)$ is the $\prec-$ maximal summand in $m_{i} e_{k}$ whose existence is guaranteed by Lemma 4.2 .

Proof. By the definition of $m_{i}^{(k)}, \pi\left(m_{i}^{(k)}\right)=\pi\left(m_{i}\right)$ and $\lambda\left(m_{i}^{(k)}\right)=\lambda\left(m_{i}\right)+\delta^{(k)}$, where $\delta^{(k)}=(1, \ldots, 1,0, \ldots, 0)$ ( $k$ ones, $n-k$ zeroes $)$. Using this with the definition of $\prec$ shows that $m_{1} \prec m_{2} \Longrightarrow m_{1}^{(k)} \prec m_{2}^{(k)}$.

Corollary 3.4. Let $m \in P_{n}$ be a monomial, $\pi=\pi(m)$ its index permutation, and $\mu=\mu(m)$ the complementary partition defined in Subsection 3.2. Let $S$ be the set of monomials which appear (with nonzero coefficient) in the expansion of $a_{\pi} \cdot e_{\mu}$. Then:

(1) $m \in S$.

(2) $m^{\prime} \in S$ and $m^{\prime} \neq m \Longrightarrow m^{\prime} \prec m$.

Proof. This is proved by iterative applications of Lemma 3.2, using $\pi(m)=\pi\left(a_{\pi(m)}\right)$ and Lemma 3.3.

A straightening algorithm follows.

Straightening algorithm. For a monomial $m \in P_{n}$, let $\pi=\pi(m)$ be its index permutation, $a_{\pi}$ the corresponding descent basis element, and $\mu=\mu(m)$ the corresponding complementary partition. Write (by Corollary 3.4)

$$
m=a_{\pi} \cdot e_{\mu}-\Sigma,
$$

where $\Sigma$ is a sum of monomials $m^{\prime} \prec m$. Repeat the process for each $m^{\prime}$.

Example. Let $m=x_{1}^{2} x_{2} x_{3} \in P_{3}$. Then $\pi(m)=123, a_{\pi(m)}=1$ and $\mu(m)=(3,1)$. Hence

$$
x_{1}^{2} x_{2} x_{3}=1 \cdot e_{(3,1)}-\Sigma=\left(x_{1} x_{2} x_{3}\right)\left(x_{1}+x_{2}+x_{3}\right)-\Sigma,
$$

so $\Sigma=x_{1} x_{2}^{2} x_{3}+x_{1} x_{2} x_{3}^{2}$. For the first summand $\pi\left(x_{1} x_{2}^{2} x_{3}\right)=213$ and $\mu\left(x_{1} x_{2}^{2} x_{3}\right)=$ (3). Indeed $x_{1} x_{2}^{2} x_{3}=a_{213} \cdot e_{3}=x_{2} \cdot\left(x_{1} x_{2} x_{3}\right)$. Similarly, for the second summand $\pi\left(x_{1} x_{2} x_{3}^{2}\right)=312$ and $\mu\left(x_{1} x_{2} x_{3}^{2}\right)=(3)$, and $x_{1} x_{2} x_{3}^{2}=a_{312} \cdot e_{3}=x_{3} \cdot\left(x_{1} x_{2} x_{3}\right)$. We have obtained

$$
x_{1}^{2} x_{2} x_{3}=a_{123} \cdot e_{(3,1)}-a_{213} \cdot e_{3}-a_{312} \cdot e_{3} .
$$

This algorithm implies

Lemma 3.5 (Straightening Lemma). Each monomial $m \in P_{n}$ has an expression

$$
m=e_{\mu(m)} \cdot a_{\pi(m)}+\sum_{m^{\prime} \prec m} n_{m^{\prime}, m} e_{\mu\left(m^{\prime}\right)} \cdot a_{\pi\left(m^{\prime}\right)},
$$

where $n_{m^{\prime}, m}$ are integers.

The following corollary appears in [4.

Corollary 3.6. The set $\left\{a_{\pi}+I_{n} \mid \pi \in S_{n}\right\}$ forms a basis for the coinvariant algebra $P_{n} / I_{n}$.

Proof. By Lemma 3.5, $\left\{a_{\pi}+I_{n} \mid \pi \in S_{n}\right\}$ spans $P_{n} / I_{n}$ as a vector space over $\mathbf{Q}$. Since $\operatorname{dim}\left(P_{n} / I_{n}\right)=\left|S_{n}\right|[21, \S 3.6]$, this is a basis. 
3.5. Descent representations. Let $\lambda=\left(\lambda_{1}, \ldots, \lambda_{n}\right)$ be a partition with at most $n$ parts. Let $P_{\lambda}^{\unlhd}$ be the subspace of the polynomial ring $P_{n}=\mathbf{Q}\left[x_{1}, \ldots, x_{n}\right]$ spanned by all monomials whose exponent partition is dominated by $\lambda$ :

$$
P_{\lambda}^{\unlhd}:=\operatorname{span}_{\mathbf{Q}}\{m \mid \lambda(m) \unlhd \lambda\} .
$$

Similarly, define $P_{\lambda}^{\triangleleft}$ by strict dominance:

$$
P_{\lambda}^{\triangleleft}:=\operatorname{span}_{\mathbf{Q}}\{m \mid \lambda(m) \triangleleft \lambda\} .
$$

These subspaces are $S_{n}$-modules consisting of homogeneous polynomials of degree $k=|\lambda|$.

Now consider the canonical projection of $P_{n}$ onto the coinvariant algebra

$$
\psi: P_{n} \longrightarrow P_{n} / I_{n} \text {. }
$$

Define $R_{\lambda}$ to be a quotient of images under this map:

$$
R_{\lambda}:=\psi\left(P_{\lambda}^{\triangleleft}\right) / \psi\left(P_{\lambda}^{\triangleleft}\right) \text {. }
$$

Then $R_{\lambda}$ is also an $S_{n}$-module. We will show that every homogeneous component of the coinvariant algebra may be decomposed into a direct sum of certain $R_{\lambda}$ 's.

For any subset $S \subseteq\{1, \ldots, n\}$ define a partition

$$
\lambda_{S}:=\left(\lambda_{1}, \ldots, \lambda_{n}\right)
$$

by

$$
\lambda_{i}:=|S \cap\{i, \ldots, n\}| \quad(1 \leq i \leq n) .
$$

For example, for any $\pi \in S_{n}$

$$
\lambda_{\operatorname{Des}(\pi)}=\left(d_{1}(\pi), \ldots, d_{n}(\pi)\right) .
$$

Hence we have the following claim.

Claim 3.7. For any permutation $\pi \in S_{n}$

$$
\lambda\left(a_{\pi}\right)=\lambda_{\operatorname{Des}(\pi)} .
$$

The Straightening Lemma (Lemma 3.5) implies

Lemma 3.8. For any two permutations $\tau$ and $\pi$ in $S_{n}$, the action of $\tau$ on the monomial $a_{\pi} \in P_{n}$ has the expression

$$
\tau\left(a_{\pi}\right)=\sum_{\left\{w \in S_{n} \mid \lambda\left(a_{w}\right) \unlhd \lambda\left(a_{\pi}\right)\right\}} n_{w} a_{w}+p,
$$

where $n_{w} \in \boldsymbol{Z}$ and $p \in I_{n}$.

Proof. Apply the Straightening Lemma (Lemma 3.5) to $m=\tau\left(a_{\pi}\right)$. Note that $e_{\mu\left(m^{\prime}\right)} \notin I_{n}$ iff $\mu\left(m^{\prime}\right)=\emptyset$, and then $m^{\prime}=a_{\pi\left(m^{\prime}\right)}$. Denoting $w:=\pi\left(m^{\prime}\right)$ we get $m^{\prime} \preceq m \Longrightarrow \lambda\left(a_{w}\right)=\lambda\left(m^{\prime}\right) \unlhd \lambda(m)=\lambda\left(\tau\left(a_{\pi}\right)\right)=\lambda\left(a_{\pi}\right)$.

Another description of the $S_{n}$-module $\psi\left(P_{\lambda}^{\unlhd}\right)$ follows.

Lemma 3.9. For any partition $\lambda$

$$
\psi\left(P_{\lambda}^{\unlhd}\right)=\operatorname{span}_{\mathbf{Q}}\left\{a_{\pi}+I_{n} \mid \pi \in S_{n}, \lambda\left(a_{\pi}\right) \unlhd \lambda\right\} .
$$

Proof. Clearly, for any $\pi \in S_{n}$ with $\lambda\left(a_{\pi}\right) \unlhd \lambda, a_{\pi}+I_{n}=\psi\left(a_{\pi}\right) \in \psi\left(P_{\lambda}^{\unlhd}\right)$. The opposite inclusion follows from Lemma [3.5. 
Let

and

$$
J_{\lambda}^{\unlhd}:=\operatorname{span}_{\mathbf{Q}}\left\{a_{\pi}+I_{n} \mid \pi \in S_{n}, \lambda\left(a_{\pi}\right) \unlhd \lambda\right\}
$$

By Lemma 3.9

$$
J_{\lambda}^{\triangleleft}:=\operatorname{span}_{\mathbf{Q}}\left\{a_{\pi}+I_{n} \mid \pi \in S_{n}, \lambda\left(a_{\pi}\right) \triangleleft \lambda\right\} .
$$

Hence

$$
R_{\lambda}=J \frac{\triangleleft}{\lambda} / J_{\lambda}^{\triangleleft}
$$

Corollary 3.10. The following conditions on a partition $\lambda=\left(\lambda_{1}, \ldots, \lambda_{n}\right)$ are equivalent:

(1) $R_{\lambda} \neq 0$.

(2) $\lambda=\lambda\left(a_{\pi}\right)$ for some $\pi \in S_{n}$.

(3) $\lambda=\lambda_{S}$ for some $S \subseteq[n-1]$.

(4) The difference between consecutive parts of $\lambda$ is either 0 or 1 , i.e. (denoting $\lambda_{n+1}:=0$ ),

$$
\lambda_{i}-\lambda_{i+1} \in\{0,1\} \quad(1 \leq i \leq n) .
$$

Proof. $R_{\lambda} \neq 0$ if and only if $J_{\lambda}^{\triangleleft} \neq J_{\lambda}^{\triangleleft}$. This happens if and only if there exists a permutation $\pi \in S_{n}$ for which $\lambda\left(a_{\pi}\right)=\lambda$ (since, by Corollary 3.6, the various $a_{\pi}+I_{n}$ are linearly independent). By Claim 3.7 $\lambda\left(a_{\pi}\right)=\lambda_{S}$ for $S=\operatorname{Des}(\pi)$. Conversely, any subset of $[n-1]$ is a descent set for an appropriate permutation. This shows the equivalence of the first three conditions. The equivalence of (3) and (4) follows easily from the definition of $\lambda_{S}$.

From now on denote $R_{S}:=R_{\lambda_{S}}$. For $\pi \in S_{n}$, let $\bar{a}_{\pi}$ be the image of the descent basis element $a_{\pi}+I_{n} \in J \bar{\lambda}_{S}$ in the quotient $R_{S}$, where $S:=\operatorname{Des}(\pi)$.

Corollary 3.11. For any subset $S \subseteq[n-1]$, the set

$$
\left\{\bar{a}_{\pi} \mid \pi \in S_{n}, \operatorname{Des}(\pi)=S\right\}
$$

forms a basis of $R_{S}$.

Proof. Follows by elementary linear algebra from Corollary 3.6. Claim 3.7 and the definitions of $J \frac{\unlhd}{S}, J_{S}^{\triangleleft}$ and $\bar{a}_{\pi}$.

Recall the notation $R_{k}$ for the $k$-th homogeneous component of the coinvariant algebra $P_{n} / I_{n}$.

Theorem 3.12. For every $0 \leq k \leq\left(\begin{array}{l}n \\ 2\end{array}\right)$,

$$
R_{k} \cong \bigoplus_{S} R_{S}
$$

as $S_{n}$-modules, where the sum is over all subsets $S \subseteq[n-1]$ such that $\sum_{i \in S} i=k$.

Proof. Note that $a_{\pi}+I_{n} \in R_{k} \Longleftrightarrow m a j(\pi)=k$. It follows, by Corollary 3.6 that $\left\{a_{\pi}+I_{n} \mid \operatorname{maj}(\pi)=k\right\}$ is a basis for $R_{k}$, so that, by Corollary $3.11 R_{k} \cong \bigoplus_{S} R_{S}$ (sum over all $S \subseteq[n-1]$ with $\sum_{i \in S} i=k$ ) as vector spaces over $\mathbf{Q}$. By Maschke's Theorem, if $V$ is a finite-dimensional $G$-module for a finite group $G$ (over a field of characteristic zero) and $W \subseteq V$ is a $G$-submodule, then $V \cong W \oplus(V / W)$ as $G$-modules. Apply this to the poset

$$
\left\{J_{S}^{\unlhd} \mid S \subseteq[n-1], \sum_{i \in S} i=k\right\}
$$


ordered by dominance order on the partitions $\lambda_{S}$. Using the definition of $R_{S}$, we get by induction on the poset the required isomorphism.

\section{Decomposition of Descent Representations}

In this section we refine the Lusztig-Stanley Theorem.

Theorem 4.1. For any subset $S \subseteq[n-1]$ and partition $\mu \vdash n$, the multiplicity in $R_{S}$ of the irreducible $S_{n}$-representation corresponding to $\mu$ is

$$
m_{S, \mu}:=|\{T \in S Y T(\mu) \mid \operatorname{Des}(T)=S\}| .
$$

The proof applies an argument of Stanley (cf. 28, Theorem 8.8] and references therein), using symmetric functions. Its use is possible here due to the Straightening Lemma. A key lemma in the proof is a multivariate version of Stanley's formula.

4.1. A multivariate version of Stanley's Formula. In this subsection we state and prove a multivariate version of a well known formula of Stanley for the principal specialization of a Schur function.

Recall that $s_{\lambda}\left(x_{1}, \ldots, x_{n}\right)$ denotes the Schur function corresponding to a partition $\lambda$ of $n$. Then

Proposition 4.2 ([36, Prop. 7.19.11]). If $\lambda$ is a partition of $n$, then

$$
s_{\lambda}\left(1, q, q^{2}, \ldots\right)=\frac{\sum_{T \in S Y T(\lambda)} q^{\operatorname{maj}(T)}}{(1-q)\left(1-q^{2}\right) \cdots\left(1-q^{n}\right)},
$$

where $T$ runs through all standard Young tableaux of shape $\lambda$.

Define $\mathbf{Q}\left[\left[z_{1}, z_{1} z_{2}, \ldots\right]\right]$ to be the ring of formal power series in countably many variables $z_{1}, z_{1} z_{2}, \ldots, z_{1} z_{2} \cdots z_{k}, \ldots$; a linear basis for it consists of the monomials $z^{\lambda}:=z_{1}^{\lambda_{1}} \cdots z_{n}^{\lambda_{k}}$ for all partitions $\lambda=\left(\lambda_{1}, \ldots, \lambda_{k}\right)\left(\lambda_{1} \geq \cdots \geq \lambda_{k} \geq 0\right)$. Let $\mathbf{Q}\left[\left[q_{1}, q_{1} q_{2}, \ldots\right]\right]$ be similarly defined.

Let $\iota: \mathbf{Q}\left[\left[z_{1}, z_{1} z_{2}, \ldots\right]\right] \longrightarrow \mathbf{Q}\left[\left[q_{1}, q_{1} q_{2}, \ldots\right]\right]$ be defined by

$$
\iota\left(z^{\lambda}\right):=q^{\lambda^{\prime}} \quad(\forall \lambda)
$$

where $\lambda^{\prime}$ is the partition conjugate to $\lambda$. Extend $\iota$ by linearity. Note that $\iota$ is not a ring homomorphism.

For any standard Young tableau $T$ define

$$
d_{i}(T):=|\{j \geq i \mid j \in \operatorname{Des}(T)\}| \quad(1 \leq i \leq n),
$$

where $\operatorname{Des}(T)$ is the set of all descents in $T$ (as in Subsection [2.3).

Lemma 4.3 (Multivariate Formula). If $\lambda$ is a partition of $n$, then

$$
\iota\left[s_{\lambda}\left(1, z_{1}, z_{1} z_{2}, \ldots, z_{1} z_{2} \cdots z_{i}, \ldots\right)\right]=\frac{\sum_{T \in S Y T(\lambda)} \prod_{i=1}^{n} q_{i}^{d_{i}(T)}}{\prod_{i=1}^{n}\left(1-q_{1} q_{2} \cdots q_{i}\right)},
$$

where $T$ runs through all standard Young tableaux of shape $\lambda$.

Note that Proposition 4.2 is the special case obtained by substituting $q_{1}=q_{2}=$ $\ldots=q_{n}=q$. Lemma 4.3] is actually implicit in [32. p. 27] (formula (18), within the proof of Theorem 9.1), in the general context of $(P, \omega)$-partitions. The fact that the LHS there is equal to the LHS above is part of the following proof, which will later be adapted to prove a $B$-analogue (Lemma 5.10 below). The key bijection is basically the one used in [38, Lemma 3.1]. 
Proof. By Claim 2.1

$$
s_{\lambda}\left(x_{1}, x_{2}, \ldots\right)=\sum_{\hat{T} \in R S S Y T(\lambda)} \prod_{i=1}^{\infty} x_{i}^{m_{i}(\hat{T})},
$$

where $\hat{T}$ runs through all reverse semi-standard Young tableaux of shape $\lambda$, and

$$
m_{i}(\hat{T}):=\mid\{\text { cells in } \hat{T} \text { with entry } i\} \mid \quad(\forall i \geq 1) .
$$

Letting

$$
x_{1}=1
$$

and

we get

$$
x_{i}=z_{1} z_{2} \cdots z_{i-1} \quad(i \geq 2)
$$

$$
s_{\lambda}\left(1, z_{1}, z_{1} z_{2}, \ldots\right)=\sum_{\hat{T} \in R S S Y T(\lambda)} \prod_{i=1}^{\infty} z_{i}^{m>i(\hat{T})}
$$

where

$$
m_{>i}(\hat{T}):=\mid\{\text { cells in } \hat{T} \text { with entry }>i\} \mid \quad(\forall i)
$$

Of course,

$$
m_{>0}(\hat{T})=n
$$

Let $\mu(\hat{T})$ be the vector $\left(m_{>1}(\hat{T}), m_{>2}(\hat{T}), \ldots\right)$. Then $\mu(\hat{T})$ is a partition with largest part at most $n$. Note that the conjugate partition is

$$
\mu(\hat{T})^{\prime}=\left(\hat{T}_{1}-1, \hat{T}_{2}-1, \ldots, \hat{T}_{n}-1\right),
$$

where $\hat{T}_{1}, \ldots, \hat{T}_{n}$ are the entries of $\hat{T}$ in weakly decreasing order. Thus

$$
\iota\left[s_{\lambda}\left(1, z_{1}, z_{1} z_{2}, \ldots\right)\right]=\sum_{\hat{T} \in R S S Y T(\lambda)} \prod_{i=1}^{n} q_{i}^{\hat{T}_{i}-1} .
$$

Recall that $S Y T(\lambda)$ and $R S S Y T(\lambda)$ are the sets of standard Young tableaux and reverse semi-standard Young tableaux of shape $\lambda$, respectively. For any $\lambda \vdash n$ define a map

$$
\phi_{\lambda}: \operatorname{RSSYT}(\lambda) \longrightarrow S Y T(\lambda) \times \boldsymbol{N}^{n}
$$

by

$$
\phi_{\lambda}(\hat{T}):=(T, \Delta)
$$

where, for $\hat{T} \in \operatorname{RSSYT}(\lambda), T$ is a standard Young tableau of shape $\lambda$ and $\Delta=$ $\left(\Delta_{1}, \ldots, \Delta_{n}\right)$ is a sequence of nonnegative integers, defined as follows:

(1) Let $\left(\hat{T}_{1}, \ldots, \hat{T}_{n}\right)$ be the vector of entries of $\hat{T}$, in weakly decreasing order. Then $T$ is the standard Young tableau, of the same shape as $\hat{T}$, having entry $i(1 \leq i \leq n)$ in the same cell in which $\hat{T}$ has entry $\hat{T}_{i}$. If some of the entries of $\hat{T}$ are equal, then they necessarily belong to distinct columns, and the corresponding entries of $T$ are then chosen increasing from left to right (i.e., with increasing column indices).

(2) Define

$$
\Delta_{i}:=\hat{T}_{i}-d_{i}(T)-\hat{T}_{i+1}+d_{i+1}(T) \quad(1 \leq i \leq n),
$$

where, by convention, $\hat{T}_{n+1}:=1$ and $d_{n+1}(T):=0$. 
Example. Let $\lambda=(3,2,2)$ and

$$
\hat{T}=\begin{array}{rrr}
7 & 4 & 4 \\
4 & 2 \\
3 & 1
\end{array} \quad \in \operatorname{RSSYT}(\lambda) .
$$

Computing $\phi_{\lambda}(\hat{T})=(T, \Delta)$, the first step yields

$$
T=\begin{array}{lll}
1 & 3 & 4 \\
2 & 6 \\
5 & 7
\end{array} \quad \in S Y T(\lambda),
$$

so that $\operatorname{Des}(T)=\{1,4,6\}$. Therefore, $\left(\hat{T}_{1}-d_{1}(T), \ldots, \hat{T}_{7}-d_{7}(T)\right)=(4,2,2,2,2,1,1)$ and $\Delta=(2,0,0,0,1,0,0)$.

The following claim is easy to verify.

Claim 4.4. (1) $\phi_{\lambda}$ is a bijection.

(2) If $\left(\hat{T}_{1}, \ldots, \hat{T}_{n}\right)$ is the vector of entries of $\hat{T}$, in weakly decreasing order, and $\phi_{\lambda}(\hat{T})=(T, \Delta)$, then

$$
\hat{T}_{i}-1=d_{i}(T)+\sum_{j \geq i} \Delta_{j} \quad(1 \leq i \leq n) .
$$

By Claim 4.4, for any $\hat{T} \in R S S Y T(\lambda)$

$$
\prod_{i=1}^{n} q_{i}^{\hat{T}_{i}-1}=\prod_{i=1}^{n} q_{i}^{d_{i}(T)} \cdot \prod_{j=1}^{n}\left(q_{1} \cdots q_{j}\right)^{\Delta_{j}}
$$

where $(T, \Delta)=\phi_{\lambda}(\hat{T})$.

Substituting (4.3) into (4.2) we get the formula in the statement of Lemma 4.3

4.2. Proof of Theorem 4.1. For a permutation $\tau \in S_{n}$ let the (graded) trace of its action on the polynomial ring $P_{n}$ be

$$
\operatorname{Tr}_{P_{n}}(\tau):=\sum_{m}\langle\tau(m), m\rangle \cdot \bar{q}^{\lambda(m)},
$$

where the sum is over all monomials $m$ in $P_{n}, \lambda(m)$ is the exponent partition of the monomial $m$, and the inner product is such that the set of all monomials is an orthonormal basis for $P_{n}$. In particular, $\langle\tau(m), m\rangle \in\{0,1\}\left(\forall \tau \in S_{n}\right)$.

Claim 4.5. If $\tau \in S_{n}$ is of cycle type $\mu$, then the trace of its action on $P_{n}$ is

$$
\operatorname{Tr}_{P_{n}}(\tau)=\iota\left[p_{\mu}\left(1, z_{1}, z_{1} z_{2}, \ldots\right)\right],
$$

where $p_{\mu}$ is the power sum symmetric function corresponding to $\mu$, and $z_{1}, z_{2}, \ldots$ are independent variables.

Proof. For a monomial $m$ in $P_{n},\langle\tau(m), m\rangle=1$ (i.e., $\tau(m)=m$ ) if and only if, for each cycle of $\tau$, the variables $x_{i}$ with indices in that cycle all have the same exponent in $m$. If $\mu=\left(\mu_{1}, \ldots, \mu_{t}\right)$ is the cycle type of $\tau$ we thus obtain, for each sequence $\left(e_{1}, \ldots, e_{t}\right) \in \boldsymbol{N}^{t}$, a unique monomial $m$ with $\tau(m)=m$ and $e_{j}$ as the 
common exponent for cycle number $j(1 \leq j \leq t)$. Thus $\lambda(m)$ consists of $\mu_{j}$ copies of $e_{j}(1 \leq j \leq t)$, reordered, and

$$
\iota^{-1}\left[\bar{q}^{\lambda(m)}\right]=\bar{z}^{\lambda(m)^{\prime}}=\prod_{j=1}^{t}\left(z_{1} \cdots z_{e_{j}}\right)^{\mu_{j}} .
$$

Summing over all choices of $e_{1}, \ldots, e_{t}$ gives $p_{\mu}\left(1, z_{1}, z_{1} z_{2}, \ldots\right)$.

Recall that for the coinvariant algebra $P_{n} / I_{n}$ we have the descent basis $\left\{a_{\pi}+\right.$ $\left.I_{n} \mid \pi \in S_{n}\right\}$. Define, for $\tau \in S_{n}$,

$$
\operatorname{Tr}_{P_{n} / I_{n}}(\tau):=\sum_{\pi \in S_{n}}\left\langle\tau\left(a_{\pi}+I_{n}\right), a_{\pi}+I_{n}\right\rangle \cdot \bar{q}^{\lambda\left(a_{\pi}\right)},
$$

where the inner product is such that the descent basis is orthonormal.

From the Straightening Lemma (Lemma 3.5) it follows that

Claim 4.6. For every $n \geq 1$ and every $\tau \in S_{n}$

$$
\operatorname{Tr}_{P_{n}}(\tau)=\operatorname{Tr}_{P_{n} / I_{n}}(\tau) \cdot \sum_{\lambda} \bar{q}^{\lambda}
$$

where the sum is over all partitions $\lambda$ with at most $n$ parts.

Proof. Replace the monomial basis of $P_{n}$ by the homogeneous polynomial basis $\left\{a_{\pi} e_{\mu} \mid \pi \in S_{n}, \mu\right.$ is a partition with largest part at most $\left.n\right\}$. The trace $\operatorname{Tr}_{P_{n}}(\tau)$ is not changed, provided that we now use the inner product for which the new basis is orthonormal. Note that $\tau\left(e_{\mu}\right)=e_{\mu}\left(\forall \tau \in S_{n}\right)$. Also note that, by the Straightening Lemma, the $\prec$-maximal monomial $m$ in $a_{\pi} e_{\mu}$ has an exponent partition $\lambda(m)=$ $\lambda\left(a_{\pi}\right)+\mu^{\prime}$, where addition of partitions is componentwise.

\section{Observation 4.7.}

$$
\sum_{\lambda} \bar{q}^{\lambda}=\prod_{i=1}^{n} \frac{1}{1-q_{1} \cdots q_{i}}
$$

where the sum is over all partitions $\lambda$ with at most $n$ parts.

Using the Frobenius formula, Lemma 4.3 (the multivariate version of Stanley's formula) and Claim 4.5 we obtain for every permutation $\tau \in S_{n}$ of cycle type $\mu$ :

$$
\begin{aligned}
\operatorname{Tr}_{P_{n}}(\tau) & =\iota\left[p_{\mu}\left(1, z_{1}, z_{1} z_{2}, \ldots\right)\right]=\sum_{\lambda \vdash n} \chi_{\mu}^{\lambda} \iota\left[s_{\lambda}\left(1, z_{1}, z_{1} z_{2}, \ldots\right)\right] \\
& =\sum_{\lambda \vdash n} \chi_{\mu}^{\lambda} \cdot \frac{\sum_{T \in S Y T(\lambda)} \prod_{i=1}^{n} q_{i}^{d_{i}(T)}}{\prod_{i=1}^{n}\left(1-q_{1} q_{2} \cdots q_{i}\right)} .
\end{aligned}
$$

By Claim 4.6 and Observation 4.7 we now get

$$
\operatorname{Tr}_{P_{n} / I_{n}}(\tau)=\sum_{\lambda \vdash n} \chi_{\mu}^{\lambda} \sum_{T \in S Y T(\lambda)} \prod_{i=1}^{n} q_{i}^{d_{i}(T)} .
$$

We conclude that the graded multiplicity in $P_{n} / I_{n}$ of the irreducible $S_{n}$-representation corresponding to $\lambda$ is

$$
\sum_{T \in S Y T(\lambda)} \prod_{i=1}^{n} q_{i}^{d_{i}(T)}=\sum_{T \in S Y T(\lambda)} \bar{q}^{\lambda_{\operatorname{Des}(T)}} .
$$


Consider now the claim of Theorem 3.12 Its proof shows that

$$
P_{n} / I_{n} \cong \bigoplus_{S \subseteq[n-1]} R_{S}
$$

actually holds as an isomorphism of graded $S_{n}$-modules. By Corollary 3.11 and Claim 3.7, $R_{S}$ is the homogeneous component of multi-degree $\lambda_{S}$ in $P_{n} / I_{n}$. It thus follows that the multiplicity in $R_{S}$ of the irreducible $S_{n}$-representation corresponding to $\lambda$ is

$$
|\{T \in S Y T(\lambda) \mid \operatorname{Des}(T)=S\}|,
$$

and the proof of Theorem 4.1 is complete.

\section{Descent REpresentations For type $B$}

In this section we give $B$-analogues of the concepts and results in Sections 3 and 4 .

5.1. The signed descent basis. For any signed permutation $\sigma \in B_{n}$ let

$$
\operatorname{Des}(\sigma):=\{i \in[n-1]: \sigma(i)>\sigma(i+1)\}
$$

be the set of descents in $\sigma$ with respect to the standard linear order on the integers, and let

$$
d_{i}(\sigma):=|\{j \in \operatorname{Des}(\sigma): j \geq i\}| \quad(1 \leq i \leq n)
$$

be the number of descents in $\sigma$ from position $i$ on.

Also let

$$
\varepsilon_{i}(\sigma):= \begin{cases}1, & \text { if } \sigma(i)<0 \\ 0, & \text { otherwise }\end{cases}
$$

and

Note that

$$
f_{i}(\sigma):=2 d_{i}(\sigma)+\varepsilon_{i}(\sigma) .
$$

$$
\sum_{i=1}^{n} f_{i}(\sigma)=f m a j(\sigma) .
$$

Finally, associate to $\sigma$ the monomial

$$
b_{\sigma}:=\prod_{i=1}^{n} x_{|\sigma(i)|}^{f_{i}(\sigma)} .
$$

We will show that the set $\left\{b_{\sigma}+I_{n}^{B} \mid \sigma \in B_{n}\right\}$ forms a linear basis for the coinvariant algebra of type $B$. We call it the signed descent basis.

5.2. Straightening. The signed index permutation of a monomial $m=\prod_{i=1}^{n} x_{i}^{p_{i}} \in$ $P_{n}$ is the unique signed permutation $\sigma=\sigma(m) \in B_{n}$ such that

$$
\begin{gathered}
p_{|\sigma(i)|} \geq p_{|\sigma(i+1)|} \quad(1 \leq i<n), \\
p_{|\sigma(i)|}=p_{|\sigma(i+1)|} \Longrightarrow \sigma(i)<\sigma(i+1)
\end{gathered}
$$

and

$$
p_{|\sigma(i)|} \equiv 0(\bmod 2) \Longleftrightarrow \sigma(i)>0 .
$$

In other words, $\sigma$ reorders the variables $x_{i}$ as does the corresponding index permutation of type $A$ (see Subsection 3.2), after attaching a minus sign to (indices of) variables with odd exponents. Note that this reverses the order of "negative" indices. 
Example. $m=x_{1}^{2} x_{2}^{3} x_{3}^{2} x_{5} x_{6}^{3}$ and $n=7$. Then $m=x_{2}^{3} x_{6}^{3} x_{1}^{2} x_{3}^{2} x_{5}^{1} x_{4}^{0} x_{7}^{0}$ and $\sigma(m)=$ $[-6,-2,1,3,-5,4,7] \in B_{7}$.

Let $m=\prod_{i=1}^{n} x_{i}^{p_{i}}$ be a monomial in $P_{n}, \sigma=\sigma(m)$ its signed index permutation, and $b_{\sigma}$ the corresponding signed descent basis element. In analogy with Claim 3.1 (for type $A$ ) we have

Claim 5.1. The sequence $\left(p_{|\sigma(i)|}-f_{i}(\sigma)\right)_{i=1}^{n}$ of exponents in $m / b_{\sigma}$ consists of nonnegative even integers, and is weakly decreasing:

$$
p_{|\sigma(i)|}-f_{i}(\sigma) \geq p_{|\sigma(i+1)|}-f_{i+1}(\sigma) \quad(1 \leq i<n) .
$$

Proof. By condition $(3)$ above $\varepsilon_{i}(\sigma)=0 \Longleftrightarrow p_{|\sigma(i)|} \equiv 0(\bmod 2)$. The numbers $p_{|\sigma(i)|}-f_{i}=p_{|\sigma(i)|}-\varepsilon_{i}(\sigma)-2 d_{i}(\sigma)$ are therefore even integers. Also $d_{n}(\sigma)=0$ so that $p_{|\sigma(n)|}-f_{n}=p_{|\sigma(n)|}-\varepsilon_{n}(\sigma) \geq 0$. It remains to show that the sequence is weakly decreasing.

If $\sigma(i)<\sigma(i+1)$, then $d_{i}(\sigma)=d_{i+1}(\sigma)$, and $p_{|\sigma(i)|}-\varepsilon_{i}(\sigma) \geq p_{|\sigma(i+1)|}-\varepsilon_{i+1}(\sigma)$ since their difference is an even integer $\geq-1$.

If $\sigma(i)>\sigma(i+1)$ and $\varepsilon_{i}(\sigma)=\varepsilon_{i+1}(\sigma)$, then $d_{i}(\sigma)=d_{i+1}(\sigma)+1$; so

$$
\left(p_{|\sigma(i)|}-f_{i}(\sigma)\right)-\left(p_{|\sigma(i+1)|}-f_{i+1}(\sigma)\right)=p_{|\sigma(i)|}-p_{|\sigma(i+1)|}-2 \geq 0
$$

since $p_{|\sigma(i+1)|} \equiv p_{|\sigma(i+1)|}(\bmod 2)$ and because of conditions (1) and (2) above.

Finally, if $\sigma(i)>\sigma(i+1)$ and $\varepsilon_{i}(\sigma) \neq \varepsilon_{i+1}(\sigma)$, then $\sigma(i)>0>\sigma(i+1)$ so that $\varepsilon_{i}(\sigma)=0, \varepsilon(i+1)=1$ and $d_{i}(\sigma)=d_{i+1}(\sigma)+1$. Thus

$$
\left(p_{|\sigma(i)|}-f_{i}(\sigma)\right)-\left(p_{|\sigma(i+1)|}-f_{i+1}(\sigma)\right)=p_{|\sigma(i)|}-p_{|\sigma(i+1)|}-1 \geq 0 .
$$

Denote by $\mu_{B}(m)$ the partition conjugate to $\left(\frac{p_{|\sigma(i)|}-f_{i}(\sigma)}{2}\right)_{i=1}^{n}$.

Definition. For monomials $m_{1}, m_{2} \in P_{n}$ of the same total degree, $m_{1} \prec_{B} m_{2}$ if, for every $1 \leq i \leq n$, the exponents of $x_{i}$ in $m_{1}$ and $m_{2}$ have the same parity, and also either

(1) $\lambda\left(m_{1}\right) \triangleleft \lambda\left(m_{2}\right)$ (strictly smaller in dominance order); or

(2) $\lambda\left(m_{1}\right)=\lambda\left(m_{2}\right)$ and $\operatorname{inv}\left(\pi\left(m_{1}\right)\right)>\operatorname{inv}\left(\pi\left(m_{2}\right)\right)$.

Here $\pi\left(m_{i}\right)$ is the (unsigned) index permutation of $m_{i}$ as in Subsection 3.2.

Imitating the proof of Lemma 3.5 we obtain

Corollary 5.2 (Straightening Lemma). Each monomial $m \in P_{n}$ has an expression

$$
m=e_{\mu_{B}(m)}\left(x_{1}^{2}, \ldots, x_{n}^{2}\right) \cdot b_{\sigma(m)}+\sum_{m^{\prime} \prec{ }_{B} m} n_{m^{\prime}, m} e_{\mu_{B}\left(m^{\prime}\right)}\left(x_{1}^{2}, \ldots, x_{n}^{2}\right) \cdot b_{\sigma\left(m^{\prime}\right)},
$$

where $n_{m^{\prime}, m}$ are integers.

5.3. Construction of descent representations. Recall (from Subsection 2.5) the definition of $I_{n}^{B}$.

Corollary 5.3. The set

$$
\left\{b_{\sigma}+I_{n}^{B} \mid \sigma \in B_{n}\right\}
$$

forms a basis for the coinvariant algebra $P_{n} / I_{n}^{B}$.

Proof. Similar to the proof of Corollary 3.6

Remark. A different proof of Corollary 5.3 may be obtained by combining and extending the proofs of [5. Theorems 1(3) and 2]. 
Corollary 5.4. For any pair of elements $\tau$ and $\sigma$ in $B_{n}$, the action of $\tau$ on $b_{\sigma} \in P_{n}$ has the expression

$$
\tau\left(b_{\sigma}\right)=\sum_{\left\{w \in B_{n} \mid \lambda\left(b_{w}\right) \unlhd \lambda\left(b_{\sigma}\right)\right\}} n_{w} b_{w}+p,
$$

where $n_{w} \in Z$ and $p \in I_{n}^{B}$.

Proof. The same as the proof of Lemma 3.8 .

Note that, actually, $n_{w} \neq 0$ in Corollary 5.4 only if $b_{w}$ and $b_{\sigma}$ have the same number of odd exponents. Note also that, unlike the case of type $A$ (Claim 3.7), for $\sigma \in B_{n}, \lambda\left(b_{\sigma}\right)$ and $\lambda_{D e s(\sigma)}$ are not necessarily equal. Indeed, for subsets $S_{1} \subseteq[n-1]$ and $S_{2} \subseteq[n]$ define a vector $\lambda_{S_{1}, S_{2}}$ by

$$
\lambda_{S_{1}, S_{2}}:=2 \lambda_{S_{1}}+\mathbf{1}_{S_{2}}
$$

where $\lambda_{S_{1}}$ is as in Subsection 3.5 above, $\mathbf{1}_{S_{2}} \in\{0,1\}^{n}$ is the characteristic vector of $S_{2}$, and addition is componentwise. Thus

$$
\lambda_{S_{1}, S_{2}}(i)=2 \cdot\left|\left\{j \geq i \mid j \in S_{1}\right\}\right|+\mathbf{1}_{S_{2}}(i) .
$$

It should be noted that $\lambda_{S_{1}, S_{2}}$ is not always a partition.

Claim 5.5. For any $\sigma \in B_{n}$

$$
\lambda\left(b_{\sigma}\right)=\lambda_{S_{1}, S_{2}},
$$

where $\operatorname{Des}(\sigma)=S_{1}$ and $\operatorname{Neg}(\sigma)=S_{2}$.

Now define

$$
R_{\lambda}^{B}:=\psi^{B}\left(P_{\lambda}^{\triangleleft}\right) / \psi^{B}\left(P_{\lambda}^{\triangleleft}\right),
$$

where $\psi^{B}: P_{n} \longrightarrow P_{n} / I_{n}^{B}$ is the canonical map from $P_{n}$ onto the coinvariant algebra of type $B$, and $P \unlhd, P^{\triangleleft}$ are as in Subsection 3.5.

By arguments similar to those given in the proof of Lemma 3.9, $R_{\lambda}^{B}$ may be described via the signed descent basis

$$
R_{\lambda}^{B}=J_{\lambda, B}^{\triangleleft} / J_{\lambda, B}^{\triangleleft},
$$

where

and

$$
J J_{\lambda, B}^{\unlhd}:=\operatorname{span}_{\mathbf{Q}}\left\{b_{\sigma}+I_{n}^{B} \mid \sigma \in B_{n}, \lambda\left(b_{\sigma}\right) \unlhd \lambda\right\},
$$

$$
J_{\lambda, B}^{\triangleleft}:=\operatorname{span}_{\mathbf{Q}}\left\{b_{\sigma}+I_{n}^{B} \mid \sigma \in B_{n}, \lambda\left(b_{\sigma}\right) \triangleleft \lambda\right\} .
$$

Corollary 5.6. The following conditions on a partition $\lambda=\left(\lambda_{1}, \ldots, \lambda_{n}\right)$ are equivalent:

(1) $R_{\lambda}^{B} \neq 0$.

(2) $\lambda=\lambda\left(b_{\sigma}\right)$ for some $\sigma \in B_{n}$.

(3) $\lambda=\lambda_{S_{1}, S_{2}}$ for some $S_{1} \subseteq[n-1]$ and $S_{2} \subseteq[n]$, and $\lambda_{S_{1}, S_{2}}$ is a partition.

(4) The difference between consecutive parts of $\lambda$ is either 0,1 or 2, i.e. (denoting $\lambda_{n+1}:=0$ ),

$$
\lambda_{i}-\lambda_{i+1} \in\{0,1,2\} \quad(1 \leq i \leq n) .
$$

Proof. Similar to the proof of Corollary 3.10

From now on, denote $R_{S_{1}, S_{2}}^{B}:=R_{\lambda_{S_{1}, S_{2}}}^{B}$. For $\sigma \in B_{n}$, denote by $\bar{b}_{\sigma}$ the image of the signed descent basis element $b_{\sigma}+I_{w}^{B} \in J_{\lambda_{S_{1}, S_{2}, B}}^{\unlhd}$ in $R_{S_{1}, S_{2}}^{B}$. 
Corollary 5.7. For any $S_{1} \subseteq[n-1]$ and $S_{2} \subseteq[n]$, the set

$$
\left\{\bar{b}_{\sigma} \mid \sigma \in B_{n}, \operatorname{Des}(\sigma)=S_{1}, \operatorname{Neg}(\sigma)=S_{2}\right\}
$$

forms a basis of $R_{S_{1}, S_{2}}^{B}$.

Recall the notation $R_{k}^{B}$ for the $k$-th homogeneous component of $P_{n} / I_{n}^{B}$.

Theorem 5.8. For every $0 \leq k \leq n^{2}$,

$$
R_{k}^{B} \cong \bigoplus_{S_{1}, S_{2}} R_{S_{1}, S_{2}}^{B}
$$

as $B_{n}$-modules, where the sum is over all subsets $S_{1} \subseteq[n-1]$ and $S_{2} \subseteq[n]$ such that $\lambda_{S_{1}, S_{2}}$ is a partition and

$$
2 \cdot \sum_{i \in S_{1}} i+\left|S_{2}\right|=k
$$

Proof. Similar to the proof of Theorem 3.12

5.4. Decomposition of descent representations. In this subsection we give a $B$-analogue of Theorem 4.1 .

Theorem 5.9. For any pair of subsets $S_{1} \subseteq[n-1], S_{2} \subseteq[n]$, and a bipartition $\left(\mu^{1}, \mu^{2}\right)$ of $n$, the multiplicity of the irreducible $B_{n}$-representation corresponding to $\left(\mu^{1}, \mu^{2}\right)$ in $R_{S_{1}, S_{2}}^{B}$ is

$$
m_{S_{1}, S_{2}, \mu^{1}, \mu^{2}}:=\mid\left\{T \in S Y T\left(\mu^{1}, \mu^{2}\right) \mid \operatorname{Des}(T)=S_{1} \text { and } \operatorname{Neg}(T)=S_{2}\right\} \mid .
$$

Again, we need a (type $B$ ) multivariate version of Proposition 4.2. Recall definition (4.1) of the mapping $\iota$.

Let $\left(\lambda^{1}, \lambda^{2}\right)$ be a bipartition of $n$. Recall the definitions of a standard Young tableau $T=\left(T^{1}, T^{2}\right)$ of shape $\left(\lambda^{1}, \lambda^{2}\right)$ and the sets $\operatorname{Des}(T)$ and $N e g(T)$ from Subsection 2.3. Denote the set of all standard tableaux of shape $\left(\lambda^{1}, \lambda^{2}\right)$ by $S Y T\left(\lambda^{1}, \lambda^{2}\right)$. A reverse semi-standard Young tableau of shape $\left(\lambda^{1}, \lambda^{2}\right)$ is a pair $\left(\hat{T}^{1}, \hat{T}^{2}\right)$ of reverse semi-standard Young tableaux, where: $\hat{T}^{i}$ has shape $\lambda^{i}(i=1,2)$; the entries of $\hat{T}^{i}$ are congruent to $i(\bmod 2)$; the entries in each row are weakly decreasing; and the entries in each column are strictly decreasing. Denote by $\operatorname{RSSYT}\left(\lambda^{1}, \lambda^{2}\right)$ the set of all such tableaux.

For any standard Young tableau $T=\left(T^{1}, T^{2}\right)$ of shape $\left(\lambda^{1}, \lambda^{2}\right)$ define

$$
\begin{aligned}
& d_{i}(T):=|\{j \in \operatorname{Des}(T) \mid j \geq i\}|, \\
& \varepsilon_{i}(T):= \begin{cases}1, & \text { if } i \in N \operatorname{Neg}(T), \\
0, & \text { otherwise, }\end{cases}
\end{aligned}
$$

and

$$
f_{i}(T):=2 d_{i}(T)+\varepsilon_{i}(T)
$$

Then

$$
\operatorname{fmaj}(T)=\sum_{i=1}^{n} f_{i}(T)
$$


Lemma 5.10. If $\left(\lambda^{1}, \lambda^{2}\right)$ is a bipartition of $n$, then

$$
\begin{gathered}
\iota\left[s_{\lambda^{1}}\left(1, z_{1} z_{2}, z_{1} z_{2} z_{3} z_{4}, \ldots\right) \cdot s_{\lambda^{2}}\left(z_{1}, z_{1} z_{2} z_{3}, \ldots\right)\right] \\
=\frac{\sum_{T \in S Y T\left(\lambda^{1}, \lambda^{2}\right)} \prod_{i=1}^{n} q_{i}^{f_{i}(T)}}{\prod_{i=1}^{n}\left(1-q_{1}^{2} q_{2}^{2} \cdots q_{i}^{2}\right)},
\end{gathered}
$$

where $T$ runs through all standard Young tableaux of shape $\left(\lambda^{1}, \lambda^{2}\right)$.

Proof. By Claim 2.1

$$
s_{\lambda}\left(x_{1}, x_{2}, \ldots\right)=\sum_{\hat{T} \in R S S Y T(\lambda)} \prod_{i=1}^{\infty} x_{i}^{m_{i}(\hat{T})},
$$

where $\hat{T}$ runs through all reverse semi-standard Young tableaux of shape $\lambda$, and

$$
m_{i}(\hat{T}):=\mid\{\text { cells in } \hat{T} \text { with entry } i\} \mid \quad(\forall i \geq 1) .
$$

Note that for $\left(\hat{T}^{1}, \hat{T}^{2}\right) \in \operatorname{RSSYT}\left(\lambda^{1}, \lambda^{2}\right)$ we have $\left(\hat{T}^{1}+1\right) / 2 \in R S S Y T\left(\lambda^{1}\right)$, where $\left(\hat{T}^{1}+1\right) / 2$ is obtained by replacing each entry $i$ of $\hat{T}^{1}$ by $(i+1) / 2$; and similarly $\hat{T}^{2} / 2 \in \operatorname{RSSYT}\left(\lambda^{2}\right)$. Thus

$$
s_{\lambda^{1}}\left(x_{1}, x_{2}, \ldots\right) \cdot s_{\lambda^{2}}\left(y_{1}, y_{2}, \ldots\right)=\sum_{\left(\hat{T}^{1}, \hat{T}^{2}\right) \in R S S Y T\left(\lambda^{1}, \lambda^{2}\right)} \prod_{i=1}^{\infty} x_{i}^{m_{2 i-1}\left(\hat{T}^{1}\right)} y_{i}^{m_{2 i}\left(\hat{T}^{2}\right)} .
$$

Letting

$$
x_{1}=1
$$

and

for $s_{\lambda^{1}}$, while

$$
x_{i}=z_{1} z_{2} \cdots z_{2 i-2} \quad(i \geq 2)
$$

for $s_{\lambda^{2}}$, we get

$$
y_{i}=z_{1} z_{2} \cdots z_{2 i-1} \quad(i \geq 1)
$$

$$
s_{\lambda^{1}}\left(1, z_{1} z_{2}, \ldots\right) \cdot s_{\lambda^{2}}\left(z_{1}, z_{1} z_{2} z_{3}, \ldots\right)=\sum_{\left(\hat{T}^{1}, \hat{T}^{2}\right) \in R S S Y T\left(\lambda^{1}, \lambda^{2}\right)} \prod_{i=1}^{\infty} z_{i}^{m_{>i}\left(\hat{T}^{1}, \hat{T}^{2}\right)},
$$

where

$$
m_{>i}\left(\hat{T}^{1}, \hat{T}^{2}\right):=\mid\left\{\text { cells in }\left(\hat{T}^{1}, \hat{T}^{2}\right) \text { with entry }>i\right\} \mid \quad(\forall i \geq 1) .
$$

Let $\mu\left(\hat{T}^{1}, \hat{T}^{2}\right)$ be the vector $\left(m_{>1}\left(\hat{T}^{1}, \hat{T}^{2}\right), m_{>2}\left(\hat{T}^{1}, \hat{T}^{2}\right), \ldots\right)$. Then $\mu\left(\hat{T}^{1}, \hat{T}^{2}\right)$ is a partition with largest part at most $n$. The conjugate partition is

$$
\mu\left(\hat{T}^{1}, \hat{T}^{2}\right)^{\prime}=\left(\left(\hat{T}^{1}, \hat{T}^{2}\right)_{1}-1,\left(\hat{T}^{1}, \hat{T}^{2}\right)_{2}-1, \ldots,\left(\hat{T}^{1}, \hat{T}^{2}\right)_{n}-1\right),
$$

where $\left(\hat{T}^{1}, \hat{T}^{2}\right)_{1}, \ldots,\left(\hat{T}^{1}, \hat{T}^{2}\right)_{n}$ are the entries of $\left(\hat{T}^{1}, \hat{T}^{2}\right)$ in weakly decreasing order.

Thus

$$
\iota\left[s_{\lambda^{1}}\left(1, z_{1} z_{2}, \ldots\right) \cdot s_{\lambda^{2}}\left(z_{1}, \ldots\right)\right]=\sum_{\left(\hat{T}^{1}, \hat{T}^{2}\right) \in R S S Y T\left(\lambda^{1}, \lambda^{2}\right)} \prod_{i=1}^{n} q_{i}^{\left(\hat{T}^{1}, \hat{T}^{2}\right)_{i}-1} .
$$

For any bipartition $\left(\lambda^{1}, \lambda^{2}\right)$ of $n$ define a map

$$
\phi_{\left(\lambda^{1}, \lambda^{2}\right)}: \operatorname{RSSYT}\left(\lambda^{1}, \lambda^{2}\right) \longrightarrow S Y T\left(\lambda^{1}, \lambda^{2}\right) \times \boldsymbol{N}^{n}
$$

by

$$
\phi_{\left(\lambda^{1}, \lambda^{2}\right)}\left(\hat{T}^{1}, \hat{T}^{2}\right):=\left(\left(T^{1}, T^{2}\right), \Delta\right)
$$


where, for $\left(\hat{T}^{1}, \hat{T}^{2}\right) \in R S S Y T\left(\lambda^{1}, \lambda^{2}\right),\left(T^{1}, T^{2}\right)$ is a standard Young tableau of shape $\left(\lambda^{1}, \lambda^{2}\right)$ and $\Delta=\left(\Delta_{1}, \ldots, \Delta_{n}\right)$ is a sequence of nonnegative integers, defined as follows:

(1) Let $\left(\left(\hat{T}^{1}, \hat{T}^{2}\right)_{1}, \ldots,\left(\hat{T}^{1}, \hat{T}^{2}\right)_{n}\right)$ be the vector of entries of $\left(\hat{T}^{1}, \hat{T}^{2}\right)$ in weakly decreasing order. Then $\left(T^{1}, T^{2}\right)$ is the standard Young tableau, of the same shape as $\left(\hat{T}^{1}, \hat{T}^{2}\right)$, with entry $i(1 \leq i \leq n)$ in the same cell in which $\left(\hat{T}^{1}, \hat{T}^{2}\right)$ has entry $\left(\hat{T}^{1}, \hat{T}^{2}\right)_{i}$. If some of the entries of $\left(\hat{T}^{1}, \hat{T}^{2}\right)$ are equal, then they necessarily belong to distinct columns (in the same tableau), and the corresponding entries of $\left(T^{1}, T^{2}\right)$ are then chosen as consecutive integers, increasing from left to right (i.e., with increasing column indices).

(2) Define

$$
\Delta_{i}:=\left(\left(\hat{T}^{1}, \hat{T}^{2}\right)_{i}-f_{i}\left(T^{1}, T^{2}\right)-\left(\hat{T}^{1}, \hat{T}^{2}\right)_{i+1}+f_{i+1}\left(T^{1}, T^{2}\right)\right) / 2 \quad(1 \leq i \leq n)
$$

where, by convention, $\left(\hat{T}^{1}, \hat{T}^{2}\right)_{n+1}:=1$ and $f_{n+1}:=0$.

Example. Let $\left(\lambda^{1}, \lambda^{2}\right)=((3,1),(2,1))$ be a bipartition of 7 , and let

$$
\left(\hat{T}^{1}, \hat{T}^{2}\right)=\left(\begin{array}{ccccc}
11 & 7 & 3 & 10 & 8 \\
3 & & & 2 &
\end{array}\right) \in \operatorname{RSSYT}\left(\lambda^{1}, \lambda^{2}\right)
$$

Computing $\phi_{\left(\lambda^{1}, \lambda^{2}\right)}\left(\hat{T}^{1}, \hat{T}^{2}\right)=\left(\left(T^{1}, T^{2}\right), \Delta\right)$, the first step yields

$$
\left(T^{1}, T^{2}\right)=\left(\begin{array}{cccccc}
1 & 4 & 6 & 2 & 3 \\
5 & & & 7 & 7
\end{array}\right) \in \operatorname{SYT}\left(\lambda^{1}, \lambda^{2}\right)
$$

so that $\operatorname{Des}\left(T^{1}, T^{2}\right)=\{1,4,6\}$ and $\operatorname{Neg}\left(T^{1}, T^{2}\right)=\{2,3,7\}$. Therefore

$$
\begin{gathered}
\left(f_{1}\left(T^{1}, T^{2}\right), \ldots, f_{7}\left(T^{1}, T^{2}\right)\right)=(6,5,5,4,2,2,1), \\
\left(\left(\hat{T}^{1}, \hat{T}^{2}\right)_{1}-f_{1}\left(T^{1}, T^{2}\right), \ldots,\left(\hat{T}^{1}, \hat{T}^{2}\right)_{7}-f_{7}\left(T^{1}, T^{2}\right)\right)=(5,5,3,3,1,1,1),
\end{gathered}
$$

and

$$
\Delta=(0,1,0,1,0,0,0)
$$

The following claim is easy to verify.

Claim 5.11. (1) $\phi_{\left(\lambda^{1}, \lambda^{2}\right)}$ is a bijection.

(2) If $\left(\left(\hat{T}^{1}, \hat{T}^{2}\right)_{1}, \ldots,\left(\hat{T}^{1}, \hat{T}^{2}\right)_{n}\right)$ is the vector of entries of $\left(\hat{T}^{1}, \hat{T}^{2}\right)$, in weakly decreasing order, and $\phi_{\left(\lambda^{1}, \lambda^{2}\right)}\left(\hat{T}^{1}, \hat{T}^{2}\right)=\left(\left(T^{1}, T^{2}\right), \Delta\right)$, then

$$
\left(\hat{T}^{1}, \hat{T}^{2}\right)_{i}-1=f_{i}\left(T^{1}, T^{2}\right)+\sum_{j \geq i} 2 \Delta_{j} \quad(1 \leq i \leq n) .
$$

By Claim 5.11, for any $\left(\hat{T}^{1}, \hat{T}^{2}\right) \in \operatorname{RSSYT}\left(\lambda^{1}, \lambda^{2}\right)$,

$$
\prod_{i=1}^{n} q_{i}^{\left(\hat{T}^{1}, \hat{T}^{2}\right)_{i}-1}=\prod_{i=1}^{n} q_{i}^{f_{i}\left(T^{1}, T^{2}\right)} \cdot \prod_{j=1}^{n}\left(q_{1} \cdots q_{j}\right)^{2 \Delta_{j}} .
$$

Substituting (5.2) into (5.1) we get the formula in the statement of Lemma 5.10 
5.5. Proof of Theorem 5.9. For a permutation $\tau \in B_{n}$ let the trace of its action on the polynomial ring $P_{n}$ be

$$
\operatorname{Tr}_{P_{n}}(\tau):=\sum_{m}\langle\tau(m), m\rangle \bar{q}^{\lambda(m)},
$$

where the sum is over all monomials $m$ in $P_{n}, \lambda(m)$ is the exponent partition of the monomial $m$, and the inner product is such that the set of all monomials is an orthonormal basis for $P_{n}$.

Claim 5.12. If $\tau \in B_{n}$ is of cycle type $\left(\mu^{1}, \mu^{2}\right)$, then the trace of its action on $P_{n}$ is

$$
\operatorname{Tr}_{P_{n}}(\tau)=\iota\left[p_{\mu^{1}, \mu^{2}}(\bar{x}, \bar{y})\right]
$$

where $p_{\mu_{1}, \mu_{2}}$ is as in Subsection 2.4,

$$
\bar{x}:=\left(1, z_{1} z_{2}, z_{1} z_{2} z_{3} z_{4}, \ldots\right)
$$

and

$$
\bar{y}:=\left(z_{1}, z_{1} z_{2} z_{3}, \ldots\right) .
$$

Proof. Let $\mu^{1}=\left(1^{\alpha_{1}}, 2^{\alpha_{2}}, \ldots\right)$ and $\mu^{2}=\left(1^{\beta_{1}}, 2^{\beta_{2}}, \ldots\right)$. Then $\langle\tau(m), m\rangle \neq 0$ (and is \pm 1 ) iff all variables $x_{i}$ with $i$ in the same cycle of $\tau$ have equal exponents in $m$. The sign depends on the number of negative cycles with odd exponent. Thus

$$
\begin{gathered}
\iota^{-1}\left[\operatorname{Tr}_{P_{n}}(\tau)\right]=\prod_{i \geq 1}\left(\sum_{t \geq 0}\left(z_{1} \cdots z_{t}\right)^{i}\right)^{\alpha_{i}} \cdot \prod_{j \geq 1}\left(\sum_{s \geq 0}(-1)^{s}\left(z_{1} \cdots z_{s}\right)^{j}\right)^{\beta_{j}} \\
=p_{\mu^{1}, \mu^{2}}(\bar{x}, \bar{y}) .
\end{gathered}
$$

Proof of Theorem 5.9. From the Straightening Lemma for type $B$ (Corollary 5.2) it follows that for any $\tau \in B_{n}$

$$
\operatorname{Tr}_{R_{n}^{B}}(\tau) \cdot \prod_{i=1}^{n} \frac{1}{1-\left(q_{1} \cdots q_{i}\right)^{2}}=\operatorname{Tr}_{P_{n}}(\tau) .
$$

Inserting Claim 5.12 and the Frobenius formula for type $B$ (see Subsection 2.4) we obtain

$$
\begin{gathered}
\operatorname{Tr}_{R_{n}^{B}}(\tau)=\prod_{i=1}^{n}\left[1-\left(q_{1} \cdots q_{i}\right)^{2}\right] \cdot \iota\left[p_{\mu^{1}, \mu^{2}}(\bar{x}, \bar{y})\right] \\
=\prod_{i=1}^{n}\left[1-\left(q_{1} \cdots q_{i}\right)^{2}\right] \cdot \iota\left[\sum_{\lambda^{1}, \lambda^{2}} \chi_{\mu^{1}, \mu^{2}}^{\lambda^{1}, \lambda^{2}} s_{\lambda^{1}}(\bar{x}) s_{\lambda^{2}}(\bar{y})\right] .
\end{gathered}
$$

Applying the linearity of $\iota$ and Lemma 5.10 gives

$$
\operatorname{Tr}_{R_{n}^{B}}(\tau)=\sum_{\lambda^{1}, \lambda^{2}} \chi_{\mu^{1}, \mu^{2}}^{\lambda^{1}, \lambda^{2}} \sum_{\left(T^{1}, T^{2}\right) \in S Y T\left(\lambda^{1}, \lambda^{2}\right)} \prod_{i=1}^{n} q_{i}^{f_{i}\left(T^{1}, T^{2}\right)} .
$$




\section{Combinatorial identities}

In this section we apply the above algebraic setting to obtain new combinatorial identities. The basic algebraic-combinatorial tool here is the Hilbert series of polynomial rings with respect to multi-degree rearranged into a weakly decreasing sequence (i.e., a partition). The computation of the Hilbert series follows in general the one presented in [28, $\$ 8.3$ for total degree; the major difference is the fact that total degree gives a grading of the polynomial ring, whereas rearranged multi-degree only leads to a filtration: $P_{n}=\bigcup_{\lambda} P_{\bar{\lambda}}^{\triangleleft}$ and $P_{\bar{\lambda}}^{\triangleleft} \cdot P_{\bar{\mu}}^{\unlhd} \subseteq P_{\bar{\lambda}+\mu}^{\triangleleft}$. For this reason, not every homogeneous basis for the coinvariant algebra will do: the numerators of the RHS in Theorems 6.2 and 6.5 below are generating functions for the descent basis (signed descent basis, respectively), but other bases (e.g., Schubert polynomials) have different generating functions, and are therefore not appropriate for use here. The success of the argument relies on properties of the descent basis.

6.1. Main combinatorial results. For any signed permutation $\sigma \in B_{n}$ let $d_{i}(\sigma)$, $n_{i}(\sigma)$, and $\varepsilon_{i}(\sigma)$ have the same meaning as in Subsection 1.2.1.

The main result of this section is

Theorem 6.1. Let $n \in \boldsymbol{P}$. Then

$$
\sum_{\sigma \in B_{n}} \prod_{i=1}^{n} q_{i}^{d_{i}(\sigma)+n_{i}\left(\sigma^{-1}\right)}=\sum_{\sigma \in B_{n}} \prod_{i=1}^{n} q_{i}^{2 d_{i}(\sigma)+\varepsilon_{i}(\sigma)} .
$$

Proof. Theorem 6.1 is an immediate consequence of Theorems 6.5 and 6.7 below.

For any partition $\lambda=\left(\lambda_{1}, \ldots, \lambda_{n}\right)$ with at most $n$ positive parts let

$$
m_{j}(\lambda):=\left|\left\{1 \leq i \leq n \mid \lambda_{i}=j\right\}\right| \quad(\forall j \geq 0)
$$

and let $\left(\begin{array}{c}n \\ m(\lambda)\end{array}\right)$ denote the multinomial coefficient $\left(\begin{array}{c}n \\ m_{0}(\lambda), m_{1}(\lambda), \ldots\end{array}\right)$.

By considering the Hilbert series of the polynomial ring $P_{n}$ with respect to weakly decreasing multi-degree we obtain

Theorem 6.2. Let $n \in \boldsymbol{P}$. Then

$$
\sum_{\ell(\lambda) \leq n}\left(\begin{array}{c}
n \\
\bar{m}(\lambda)
\end{array}\right) \prod_{i=1}^{n} q_{i}^{\lambda_{i}}=\frac{\sum_{\pi \in S_{n}} \prod_{i=1}^{n} q_{i}^{d_{i}(\pi)}}{\prod_{i=1}^{n}\left(1-q_{1} \cdots q_{i}\right)}
$$

in $\boldsymbol{Z}\left[\left[q_{1}, \ldots, q_{n}\right]\right]$, where the sum on the left-hand side is taken over all partitions with at most $n$ parts.

The theorem will be proved using the following lemma.

Recall from Subsection 3.2 the definitions of the index permutation $\pi(m)$ and the complementary partition $\mu(m)$ of a monomial $m \in P_{n}$.

Lemma 6.3. The mapping $m \longmapsto\left(\pi(m), \mu(m)^{\prime}\right)$ is a bijection between the set of all monomials in $P_{n}$ and the set of all pairs $(\pi, \tilde{\mu})$, where $\pi \in S_{n}$ and $\tilde{\mu}$ is a partition with at most $n$ parts.

Proof of Lemma 6.3. The indicated mapping is clearly into the claimed set, since the largest part of the complementary partition $\mu(m)$ is at most $n$. Conversely, to each pair $(\pi, \tilde{\mu})$ as in the statement of the lemma, associate the $\prec$-maximal monomial $m$ in the expansion of $a_{\pi} \cdot e_{\tilde{\mu}^{\prime}}$. Then $\pi(m)=\pi$ and $\mu(m)^{\prime}=\tilde{\mu}$. Thus the mapping is a bijection. 
Proof of Theorem 6.2. Recall the notation $\lambda(m)$ for the exponent partition of a monomial $m \in P_{n}$. For any partition with $n$ parts denote by $\bar{q}^{\lambda}$ the product $\prod_{i=1}^{n} q_{i}^{\lambda_{i}}$.

For any partition $\lambda$ with at most $n$ parts, $\left(\begin{array}{c}n \\ \bar{m}(\lambda)\end{array}\right)$ is the number of monomials in $P_{n}$ with exponent partition equal to $\lambda$. Therefore the Hilbert series of the polynomial ring $P_{n}$ by exponent partition is equal to the LHS of the theorem.

On the other hand, since $\lambda(m)=\lambda\left(a_{\pi(m)}\right)+\mu(m)^{\prime}$, using Lemma 6.3 we get

$$
\sum_{m \in P_{n}} \bar{q}^{\lambda(m)}=\sum_{m \in P_{n}} \bar{q}^{\lambda\left(a_{\pi(m)}\right)+\mu(m)^{\prime}}=\sum_{\pi \in S_{n}} \bar{q}^{\lambda\left(a_{\pi}\right)} \cdot \sum_{\mu} \bar{q}^{\mu}
$$

where $\mu$ in the last sum runs through all partitions having at most $n$ parts. By Claim 3.7 and Observation 4.7 this product is equal to the RHS of the theorem.

A well-known result, attributed by Garsia [14] to Gessel [18, follows.

Corollary 6.4. Let $n \in \boldsymbol{P}$. Then

$$
\frac{\sum_{\pi \in S_{n}} t^{\operatorname{des}(\pi)} q^{\operatorname{maj}(\pi)}}{\prod_{i=0}^{n}\left(1-t q^{i}\right)}=\sum_{r \geq 0}[r+1]_{q}^{n} t^{r}
$$

in $\boldsymbol{Z}[q][[t]]$.

Proof. Substitute, in Theorem [6.2, $q_{1}=q t$ and $q_{2}=q_{3}=\cdots=q_{n}=q$, divide both sides by $1-t$, and apply (3.1) and (3.2). We obtain

$$
\begin{gathered}
\frac{\sum_{\pi \in S_{n}} t^{\operatorname{des}(\pi)} q^{m a j(\pi)}}{\prod_{i=0}^{n}\left(1-t q^{i}\right)}=\sum_{\ell(\lambda) \leq n}\left(\begin{array}{c}
n \\
\bar{m}(\lambda)
\end{array}\right) \cdot q^{\Sigma_{i} \lambda_{i}} \cdot \frac{t^{\lambda_{1}}}{1-t} \\
=\sum_{r=0}^{\infty} \sum_{\ell(\lambda) \leq n, \lambda_{1} \leq r}\left(\begin{array}{c}
n \\
\bar{m}(\lambda)
\end{array}\right) q^{\Sigma_{i} \lambda_{i}} t^{r} .
\end{gathered}
$$

The coefficient of $t^{r}$ is

$$
\sum_{\ell(\lambda) \leq n, \lambda_{1} \leq r}\left(\begin{array}{c}
n \\
\bar{m}(\lambda)
\end{array}\right) q^{\Sigma_{i} \lambda_{i}}=\sum_{\left(\ell_{1}, \ldots, \ell_{n}\right) \in[0, r]^{n}} q^{\Sigma_{i} \ell_{i}}=\left(\sum_{j=0}^{r} q^{j}\right)^{n}=[r+1]_{q}^{n} .
$$

The same Hilbert series of $P_{n}$ may be computed in a different way, by considering the signed descent basis for the coinvariant algebra of type $B$ and applying the Straightening Lemma for this type.

Theorem 6.5. With notations as in Theorem 6.2,

$$
\sum_{\ell(\lambda) \leq n}\left(\begin{array}{c}
n \\
\bar{m}(\lambda)
\end{array}\right) \prod_{i=1}^{n} q_{i}^{\lambda_{i}}=\frac{\sum_{\sigma \in B_{n}} \prod_{i=1}^{n} q_{i}^{2 d_{i}(\sigma)+\varepsilon_{i}(\sigma)}}{\prod_{i=1}^{n}\left(1-q_{1}^{2} \cdots q_{i}^{2}\right)}
$$

in $\boldsymbol{Z}\left[\left[q_{1}, \ldots, q_{n}\right]\right]$, where the sum on the left-hand side runs through all partitions with at most $n$ parts.

Recall from Subsection 5.2 the definitions of the signed index permutation $\sigma(m) \in$ $B_{n}$ and the complementary partition $\mu_{B}(m)$ of a monomial $m \in P_{n}$. 
Lemma 6.6. The mapping $m \longmapsto\left(\sigma(m), \mu_{B}(m)^{\prime}\right)$ is a bijection between the set of all monomials in $P_{n}$ and the set of all pairs $(\sigma, \tilde{\mu})$, where $\sigma \in B_{n}$ and $\tilde{\mu}$ is a partition with at most $n$ parts.

Proof of Lemma 6.6. Similar to the proof of Lemma 6.3. Here to each pair $(\sigma, \tilde{\mu})$ associate the $\prec$-maximal monomial $m$ in $b_{\sigma} \cdot e_{\tilde{\mu}^{\prime}}\left(x_{1}^{2}, \ldots, x_{n}^{2}\right)$, and then $\sigma(m)=\sigma$ and $\mu_{B}(m)^{\prime}=\tilde{\mu}$.

Proof of Theorem 6.5. Again, the LHS of the theorem is equal to the Hilbert series of the polynomial ring $P_{n}$ by exponent partition. On the other hand, since $\lambda(m)=$ $\lambda\left(b_{\sigma(m)}\right)+2 \mu_{B}(m)^{\prime}$, Lemma 6.6 gives

$$
\sum_{m \in P_{n}} \bar{q}^{\lambda(m)}=\sum_{m \in P_{n}} \bar{q}^{\lambda\left(b_{\sigma(m)}\right)+2 \mu_{B}(m)^{\prime}}=\sum_{\sigma \in B_{n}} \bar{q}^{\lambda\left(b_{\sigma}\right)} \cdot \sum_{\mu} \bar{q}^{2 \mu}
$$

where $\mu$ in the last sum runs through all partitions having at most $n$ parts. By Claim 5.5 this product is equal to the RHS of the theorem.

Direct combinatorial arguments imply

Theorem 6.7. With notations as in Theorem 6.2,

$$
\sum_{\ell(\lambda) \leq n}\left(\begin{array}{c}
n \\
\bar{m}(\lambda)
\end{array}\right) \prod_{i=1}^{n} q_{i}^{\lambda_{i}}=\frac{\sum_{\sigma \in B_{n}} \prod_{i=1}^{n} q_{i}^{d_{i}(\sigma)+n_{i}\left(\sigma^{-1}\right)}}{\prod_{i=1}^{n}\left(1-q_{1}^{2} \cdots q_{i}^{2}\right)}
$$

in $\boldsymbol{Z}\left[\left[q_{1}, \ldots, q_{n}\right]\right]$.

Proof. Define the subset $T \subseteq B_{n}$ by

$$
T:=\left\{\pi \in B_{n}: \operatorname{des}(\pi)=0\right\} .
$$

It is clear from our definitions that $d_{i}(\sigma u)=d_{i}(u)$ and $n_{i}\left(u^{-1} \sigma^{-1}\right)=n_{i}\left(\sigma^{-1}\right)$ for all $\sigma \in T, u \in S_{n}$ and $1 \leq i \leq n$. Therefore

$$
\begin{aligned}
\sum_{\pi \in B_{n}} \prod_{i=1}^{n} q_{i}^{d_{i}(\pi)+n_{i}\left(\pi^{-1}\right)} & =\sum_{u \in S_{n}} \sum_{\sigma \in T} \prod_{i=1}^{n} q_{i}^{d_{i}(\sigma u)+n_{i}\left((\sigma u)^{-1}\right)} \\
& =\sum_{u \in S_{n}} \sum_{\sigma \in T} \prod_{i=1}^{n} q_{i}^{d_{i}(u)+n_{i}\left(\sigma^{-1}\right)} \\
& =\sum_{u \in S_{n}} \prod_{i=1}^{n} q_{i}^{d_{i}(u)} \cdot \sum_{\sigma \in T} \prod_{i=1}^{n} q_{i}^{n_{i}\left(\sigma^{-1}\right)} .
\end{aligned}
$$

An element $\sigma \in T$ is uniquely determined by the set $N e g\left(\sigma^{-1}\right)$. Hence

$$
\sum_{\sigma \in T} \prod_{i=1}^{n} q_{i}^{n_{i}\left(\sigma^{-1}\right)}=\prod_{i=1}^{n}\left(1+q_{1} \cdots q_{i}\right) .
$$

Theorem 6.2 completes the proof. 
6.2. Negative and flag statistics. In this subsection we present a central result from [1] and show that it may be obtained as a special case of results from the previous subsection.

For any $\sigma \in B_{n}$ define the negative descent multiset by

$$
N \operatorname{Des}(\sigma):=\operatorname{Des}(\sigma) \biguplus \operatorname{Neg}\left(\sigma^{-1}\right),
$$

where $\operatorname{Neg}(\sigma)$ is the set of positions of negative entries in $\sigma$, defined in Subsection 1.2.1. Note that $N \operatorname{Des}(\sigma)$ can be defined rather naturally also in purely Coxeter group theoretic terms. In fact, for $i \in[n]$ let $\eta_{i} \in B_{n}$ be defined by

$$
\eta_{i}:=[1, \ldots, i-1,-i, i+1, \ldots, n],
$$

so $\eta_{1}=s_{0}$. Then $\eta_{1}, \ldots, \eta_{n}$ are reflections of $B_{n}$ (in the Coxeter group sense; see, e.g., 21]). Clearly

$$
N \operatorname{Des}(\sigma)=\left\{i \in[n-1]: l\left(\sigma s_{i}\right)<l(\sigma)\right\} \biguplus\left\{i \in[n]: l\left(\sigma^{-1} \eta_{i}\right)<l\left(\sigma^{-1}\right)\right\} .
$$

For $\sigma \in B_{n}$ let

$$
n \operatorname{des}(\sigma):=|N \operatorname{Des}(\sigma)|, \quad \operatorname{nmaj}(\sigma):=\sum_{i \in N \operatorname{Des}(\sigma)} i .
$$

Recall the notation

$$
f \operatorname{maj}(\sigma)=2 \operatorname{maj}(\sigma)+|N e g(\sigma)|
$$

and let

$$
f \operatorname{des}(\sigma):=2 \operatorname{des}(\sigma)+\varepsilon_{1}(\sigma) .
$$

Then the two pairs of statistics (fdes, fmaj) and ( $n d e s, n m a j)$ are equidistributed over $B_{n}$.

Corollary 6.8 ([1, Corollary 4.5]). Let $n \in \boldsymbol{P}$. Then

$$
\sum_{\sigma \in B_{n}} t^{n d e s(\sigma)} q^{n m a j(\sigma)}=\sum_{\sigma \in B_{n}} t^{f d e s(\sigma)} q^{f m a j(\sigma)} .
$$

Proof. Substitute in Theorem $6.1 q_{1}=q t, q_{2}=\cdots=q_{n}=q$, and apply the identities

$$
\begin{gathered}
d_{1}(\sigma)+n_{1}\left(\sigma^{-1}\right)=n \operatorname{des}(\sigma) ; \\
\sum_{i=1}^{n}\left(d_{i}(\sigma)+n_{i}\left(\sigma^{-1}\right)\right)=n m a j(\sigma) ;
\end{gathered}
$$

and

$$
\sum_{i=1}^{n}\left(2 \cdot d_{i}(\sigma)+\varepsilon_{i}(\sigma)\right)=f m a j(\sigma)
$$

\section{ACKNOWLEDGMENTS}

This paper grew out of stimulating discussions with Dominique Foata and Ira Gessel during the conference "Classical Combinatorics" in honor of Foata's 65th birthday. The authors are indebted to Ira Gessel for the idea of using the coinvariant algebra in the study of multivariate statistics. Thanks are also due to Eli Bagno and Richard Stanley for useful comments. 


\section{REFERENCES}

[1] R. M. Adin, F. Brenti and Y. Roichman, Descent numbers and major indices for the hyperoctahedral group, Adv. Appl. Math. 27 (2001), 210-224.

[2] R. M. Adin and Y. Roichman, A flag major index for signed permutations, Proc. 11-th Conference on Formal Power Series and Algebraic Combinatorics, Universitat Politècnica de Catalunya, Barcelona, 1999, 10-17.

[3] R. M. Adin and Y. Roichman, The flag major index and group actions on polynomial rings, Europ. J. Combin. 22 (2001), 431-446. MR 2002d:05004

[4] E. E. Allen, The descent monomials and a basis for the diagonally symmetric polynomials, J. Alg. Combin. 3 (1994), 5-16. MR 95e:05121

[5] S. Ariki, T. Terasoma and H. F. Yamada, Higher Specht polynomials, Hiroshima Math. J. 27 (1997), 177-188. MR 98c:05163

[6] H. Barcelo, Young straightening in a quotient $S_{n}$-module, J. Alg. Combin. 2 (1993), 5-23. MR 94b:20016

[7] I. N. Bernstein, I. M. Gelfand and S. I. Gelfand, Schubert cells and cohomology of Schubert spaces $G / P$, Usp. Mat. Nauk. 28 (1973), 3-26. MR 55:2941

[8] A. Björner and F. Brenti, Combinatorics of Coxeter Groups, Graduate Texts in Mathematics, Springer-Verlag, to appear.

[9] F. Brenti, q-Eulerian polynomials arising from Coxeter groups, Europ. J. Combin. 15 (1994), 417-441. MR 95i:05013

[10] M. Demazure, Invariants symétriques entiers des groupes de Weyl et torsion, Invent. Math. 21 (1973), 287-301. MR 49:7268

[11] D. Foata, personal communication, July 2000.

[12] D. Foata and G. N. Han, Calcul basique des permutations signees. I. Longueur et nombre d'inversions, Adv. Appl. Math. 18 (1997), 489-509. MR 98c:05001a

[13] A. M. Garsia, Combinatorial methods in the theory of Cohen-Macaulay rings, Adv. Math. 38 (1980), 229-266. MR 82f:06002

[14] A. M. Garsia, On the "maj" and "inv" q-analogues of Eulerian polynomials, Linear and Multilinear Algebra 8 (1979/80), 21-34. MR 81h:05017

[15] A. M. Garsia and C. Procesi, On certain graded $S_{n}$-modules and the $q$-Kostka polynomials, Adv. Math. 94 (1992), 82-138. MR 93j:20030

[16] A. M. Garsia and J. Remmel, Shuffles of permutations and the Kronecker product, Graphs and Combinatorics 1 (1985), 217-263. MR 89h:05006

[17] A. M. Garsia and D. Stanton, Group actions of Stanley-Reisner rings and invariants of permutation groups, Adv. Math. 51 (1984), 107-201. MR 86f:20003

[18] I. M. Gessel, Generating functions and enumeration of sequences, Ph.D. Thesis, M.I.T., 1977.

[19] I. M. Gessel, Multipartite P-partitions and inner products of Schur functions, Contemp. Math. 34 (1984), 289-302. MR 86k:05007

[20] H. L. Hiller, Geometry of Coxeter Groups, Res. Notes in Math. 54, Pitman, Boston, 1982. MR 83h:14045

[21] J. E. Humphreys, Reflection Groups and Coxeter Groups, Cambridge Studies in Advanced Mathematics, no. 29, Cambridge Univ. Press, Cambridge, 1990. MR 92h:20002

[22] D. Kazhdan and G. Lusztig, Representations of Coxeter groups and Hecke algebras, Invent. Math. 53 (1979), 165-184. MR 81j:20066

[23] W. Kraskiewicz and J. Weyman, Algebra of coinvariants and the action of a Coxeter element, Bayreuther Math. Schriften 63 (2001), 265-284 (preprint: University of Torun, 1987). MR 2002j:20026

[24] I. G. Macdonald, Symmetric Functions and Hall Polynomials, second edition, Oxford Math. Monographs, Oxford Univ. Press, Oxford, 1995. MR 96h:05207

[25] P. A. MacMahon, Combinatory Analysis, Chelsea, New York, 1960. (Originally published in 2 vols. by Cambridge University Press, 1915-1916.) MR 25:5003

[26] H. Morita and H. F. Yamada, Higher Specht polynomials for the complex reflection group $G(r, p, n)$, Hokkaido Math. J. 27 (1998), 505-515. MR 99k:20085

[27] V. Reiner, Signed permutation statistics, Europ. J. Combin. 14 (1993), 553-567. MR 95e:05008

[28] C. Reutenauer, Free Lie Algebras, London Math. Soc. Monographs, New Series 7, Oxford Univ. Press, 1993. MR 94j:17002 
[29] B. E. Sagan, The Symmetric Group: Representations, Combinatorial Algorithms \& Symmetric Functions, Wadsworth \& Brooks/Cole, 1991. MR 93f:05102

[30] L. Solomon, The orders of the finite Chevalley groups, J. Algebra 3 (1966), 376-393. MR 33:7424

[31] T. A. Springer, A construction of representations of Weyl groups, Invent. Math. 44 (1978), 279-293. MR 58:11154

[32] R. P. Stanley, Ordered Structures and Partitions, Memoirs Amer. Math. Soc. no. 119, 1972. MR 48:10836

[33] R. P. Stanley, Invariants of finite groups and their applications to combinatorics, Bull. Amer. Math. Soc. (new series) 1 (1979), 475-511. MR 81a:20015

[34] R. P. Stanley, Some aspects of group acting on finite posets, J. Combin. Theory Ser. A 32 (1982), 132-161. MR 83d:06002

[35] R. P. Stanley, Enumerative Combinatorics, Vol. 1, Wadsworth and Brooks/Cole, Monterey, CA, 1986. MR 87j:05003

[36] R. P. Stanley, Enumerative Combinatorics, Vol. 2, Cambridge Studies in Advanced Mathematics 62, Cambridge Univ. Press, Cambridge, 1999. MR 2000k:05026

[37] R. Steinberg, On a theorem of Pittie, Topology 14 (1975), 173-177. MR 51:9101

[38] J. Stembridge, On the eigenvalues of representations of reflection groups and wreath products, Pacific J. Math. 140 (1989), 353-396. MR 91a:20022

[39] T. Terasoma and H. F. Yamada, Higher Specht polynomials for the symmetric group, Proc. Japan Acad. Ser. A Math. Sci. 69 (1993), 41-44. MR 94b:20020

Department of Mathematics and Statistics, Bar-Ilan University, Ramat-Gan 52900, ISRAEL

E-mail address: radin@math.biu.ac.il

Dipartimento di Matematica, Universitá di Roma "Tor Vergata", Via della Ricerca SCientifica, 00133 Roma, Italy

E-mail address: brenti@mat.uniroma2.it

Department of Mathematics and Statistics, Bar-Ilan University, Ramat-Gan 52900, ISRAEL

E-mail address: yuvalr@math.biu.ac.il 